\title{
Self-similarity and quasi-idempotence in neural networks and related dynamical systems
}

Ludovico Minati $^{1,2, *}$, Julia Winkel ${ }^{1,3}$, Angelo Bifone ${ }^{4}$, Paweł Oświęcimka ${ }^{2}$, Jorge Jovicich ${ }^{1}$

${ }^{1}$ Centre for Mind/Brain Science (CIMeC), University of Trento, Trento, Italy

${ }^{2}$ Complex Systems Theory Department, Institute of Nuclear Physics, Polish Academy of Sciences (IFJ-PAN), Kraków, Poland

${ }^{3}$ Dept. of Cognitive Science, University of Osnabrück, Osnabrück, Germany

${ }^{4} \mathrm{CNCS} @$ UniTn, Italian Institute of Technology (IIT), Rovereto, Italy

${ }^{*}$ Corresponding author currently at

Centre for Mind/Brain Sciences (CIMeC)

University of Trento

via delle Regole 101

38123 Mattarello TN

Italy

盗+39335486670

邫llminati@ieee.org, ludovico.minati@unitn.it

西 http://www.lminati.it

and

Complex Systems Theory Department

"H. Niewodniczański” Institute of Nuclear Physics, Polish Academy of Sciences (IFJ-PAN)

ul. Radzikowskiego 152

31-342 Kraków

Poland

焦l ludovico.minati@ifj.edu.pl 


\begin{abstract}
Self-similarity across length scales is pervasively observed in natural systems. Here, we investigate topological self-similarity in complex networks representing diverse forms of connectivity in the brain and some related dynamical systems, by considering the correlation between edges directly connecting any two nodes in a network and indirect connection between the same via all triangles spanning the rest of the network. We note that this aspect of self-similarity, which is distinct from hierarchically-nested connectivity, is closely related to idempotence of the matrix representing the graph. We introduce two measures, $t(1)$ and $t(\infty)$, which represent the element-wise correlation coefficients between the initial matrix and the ones obtained after squaring it once or infinitely many times, and term the matrices which yield large values of these parameters "quasi-idempotent". These measures delineate qualitatively-different forms of "shallow" and "deep" quasi-idempotence, which are influenced by nodal strength heterogeneity. A high degree of quasi-idempotence was observed for synchronization patterns generated by mean-field Kuramoto oscillators with noise under incomplete synchronization, electronic chaotic oscillators and dissociated neural cultures, wherein the expression of quasi-idempotence correlated strongly with network maturity. Quasi-idempotence was also detected for macro-scale brain networks representing axonal connectivity, synchronization of slow activity fluctuations during idleness and co-activation across experimental tasks, and preliminary data indicated that quasi-idempotence of structural connectivity may decrease with ageing. This initial study highlights that the form of network self-similarity indexed by quasi-idempotence is detectable in diverse dynamical systems, and draws attention to it as a basis for novel topological measures representing pattern emergence and network "collectivity".
\end{abstract}

\title{
Lead paragraph
}

A pervasive property of natural phenomena is self-similarity, namely presence of patterns or properties that recur at different spatiotemporal scales, as exemplified by the morphology of Romanesco broccoli buds. Diverse networks, including brain connectivity, are organized in hierarchies of modules which resemble their constituent elements. However, another type of selfsimilarity could also be identifiable, whereby the strength of direct links between any two nodes also reflects how closely they are connected indirectly, via the rest of the network: self-similarity not of nodes and modules, but of links and paths. We show that this notion is linked to a form of matrix idempotence, and that the brain and some related dynamical systems are indeed measurably selfsimilar in this sense.

Keywords: self-similarity, idempotence, connectome, dynamical system, collectivity 


\section{INTRODUCTION}

A distinctive feature of complex systems is that collective dynamics generate fractals, namely structural and dynamical patterns that are maintained (self-similar) across an extended scaling range. The emergence of fractals is closely linked to morphogenesis and function in diverse physical and biological systems, including networks of neurons. ${ }^{1}$ Neurophysiological recordings of cultured networks (representative of meso-scale features) as well as intact brains (macro-scale) concordantly demonstrate dynamical self-similarity, deemed to be supported by self-organized criticality and detected as power-law avalanche distribution and spectra, and invariance of non-linear time-series properties within a temporal scaling range. ${ }^{2-5}$ At the same time, certain architectural features recur across spatial scales, and are, to a varying extent, apparent in both structural (axonal) connectivity and activity synchronization (so-called functional connectivity). These features, particularly scale freeness, small-worldness, modular and hierarchical organization, altogether maximise information capacity given a budget of axonal connections and metabolic load. ${ }^{6-10}$ Even the macroscopic anatomy of the brain and its vasculature have self-similar morphology. ${ }^{11}$

The topological self-similarity of brain networks is of particular interest for understanding information segregation and integration in cognitive processes. It has been established that the hierarchical organization of cerebral cortex regions recurs at different scales as a nested interconnectivity, which is in line with the notion that complex (small-world) networks can be invariant to length-scale transformations, subject to application of an appropriate renormalization procedure to coarse-grain the system. ${ }^{6,10,12}$ However, to the authors' knowledge it remains unclear whether self-similarity is also apparent in the architecture of paths, that is, whether for adjacent nodes the strength of their direct connection resembles that of the indirect connectivity between them as implemented by paths of non-unitary length which span the rest of the network. This may be viewed as correlating the "local" and "global" connectivity between a given pair of nodes. The two aspects of topological self-similarity, namely hierarchically-nested interconnectivity vs. correspondence between direct (edges) and indirect (paths) connectivity, are conceptually related but clearly mathematically distinct.

Here, we set out to investigate self-similarity between direct and indirect connectivity, with a focus on edges vs. triangles, from the perspective of the algebraic properties of the connectivity matrix. We considered a diverse set of networks and dynamical systems, starting from synthetic graphs derived from Gaussian edge strength distributions, synchronization of mean-field Kuramoto oscillators with noise, electronic chaotic oscillators, and culminating with demonstration of self-similarity in mesoscale networks of cultured neurons and macro-scale structural and functional brain networks.

\section{RELATIONSHIP BETWEEN GRAPH SELF-SIMILARITY AND MATRIX IDEMPOTENCE}

Given a symmetric weighted undirected graph $G$, one can reveal self-similarity between edges and paths by constructing another graph $\hat{G}(l, n)$, wherein each edge represents the strength of connectivity between each node pair $(i, j)$ as implemented not directly but indirectly via all possible paths spanning $l$ nodes belonging to the rest of the network, and the calculation is iterated recursively $n$ times. For $l=1$, each edge in $\hat{G}(l, n)$ corresponds to the sum of the strengths of all triangles around the two nodes, 
each triangle being defined as the product of the two corresponding edge strengths; as exemplified in Fig. 1, given $n=1$ and network size $N$, one has

$$
\hat{g}_{i, j}=\sum_{k=1}^{N} g_{i, k} g_{k, j}
$$

This operation evidently corresponds to squaring the symmetric matrix representing the graph. For a network that has self-similar connectivity, intended as similarity between edges and paths of length $l$, one expects $G \propto \hat{G}(l, n)$, and more specifically

$$
G /\|G\| \approx \hat{G}(l, n) /\|\hat{G}(l, n)\|
$$

A matrix $\mathbf{M}$ is said to be idempotent if $\mathbf{M}=\mathbf{M}^{2}$, but for ease of explanation in this context we extend the definition to the more general case $\mathbf{M}=k \mathbf{M}^{2}$, where $k>0$ is an arbitrary scaling factor. ${ }^{13}$ While experimentally-derived graphs are unlikely to be perfectly idempotent, one can empirically assess the strength of correlation between matrix entries, which can then be taken as a measure of "quasiidempotence" (this term has been used before in reference to polynomial matrices, but defined differently). In the case of $G$ and $\hat{G}(1, n)$, quasi-idempotence in this sense represents the similarity (correlation) between direct and indirect connectivity via edges and triangles, in other words, network self-similarity across scales, intended not as hierarchies of modules and nodes, but scales of path length (namely, zero for direct edges, one for triangles). In this study, we demonstrate that networks from diverse dynamical systems including the brain are self-similar in this sense, and thus propose to consider quasi-idempotence as measure of structure in these networks.

By definition, an idempotent matrix $\mathbf{M}$ squared infinitely many times equals itself. Furthermore, as detailed in the next section, in the case of a non-pathological real-valued positive matrix $\mathbf{M}$, repeated squaring eventually leads to convergence to an idempotent matrix. ${ }^{14-16}$ Hence one can define two parameters: the linear (Pearson) correlation coefficient between off-diagonal entries of the initial and derived matrices after squaring once, or after infinitely many iterations of this operation. We refer to the former as $t(1)$ and to the latter as $t(\infty)$, and chose to quantify similarity empirically via the linear correlation coefficient as this is a bounded measure and has an immediate interpretation. Both $l(1)$ and $t(\infty)$ are by definition invariant to node permutation. In this study, we always zeroed the diagonal entries of the initial matrix (only), i.e. self-connections are disregarded, because oftentimes their definition is degenerate and corresponding measurements are problematic. Hence only off-diagonal elements were considered for determining correlation. We chose to re-normalize the matrix to unit norm at each iteration to maintain its entries in a consistent numeric range. Further, by assuming $\|\mathbf{M}\|=1$ the limit of infinitely many iterations can be approximated by stopping when

$$
\left\|\mathbf{M}-\mathbf{M}^{2}\right\|<\varepsilon,
$$

where $\varepsilon$ represents the accuracy of double-precision floating-point calculation, which implies obtaining a unit-rank matrix. ${ }^{17}$ For the matrices considered in this study, this condition was generally met after 5 to 10 iterations of squaring. Since $t(1)$ and $t(\infty)$ are relevant for undirected graphs 
(symmetric matrices), directed networks will not be considered; the present work is also not applicable to graphs that include negative weights. Notably, matrix idempotence is related to the eigenvalue spectrum, whereby according to the strict definition of idempotence the only possible eigenvalues of an idempotent matrix are 0 and 1 ; as exemplified below, this relationship could be generalizable to the case of quasi-idempotence as intended in this study. Because in this study the diagonal entries of the initial matrix are nulled, there is no immediate relationship to its positive definiteness.

Based on $\imath(1)$ and $\imath(\infty)$, three qualitatively-different situations can arise. If a graph does not possess self-similarity between edges and triangles, the corresponding matrix is distant from being idempotent, hence squaring it even once changes its structure substantially, yielding $\imath(1) \approx \imath(\infty) \approx 0$. If a graph has limited self-similar features but its global structure is not stable under repeated squaring, a "shallow" form of quasi-idempotence is present, whereby the correlation is initially high but repeated squaring eventually dissipates the initial structure or distorts it, leading to a matrix not resembling the initial one, hence $t(1)>>0$ and $t(\infty)<<\imath(1)$. If a graph has global topology close to complete selfsimilarity, the corresponding matrix already resembles the one that repeated squaring eventually converges to, hence "deep" quasi-idempotence is manifest as $t(1)>>0$ and $t(\infty) \approx t(1)$. The proposed qualitative distinction between "shallow" and "deep" forms of quasi-idempotence is orthogonal to the values of $l(1)$ and $t(\infty)$ that are actually attained, which are not only dependent on the properties of the initial matrix but also on the chosen correlation metric (e.g., linear vs. non-linear). Throughout the remainder of the study with the expression "degree of quasi-idempotence" we shall encompass both the form of quasi-idempotence according to this scheme, and its strength as represented by the numerical values of $t(1)$ and $t(\infty)$. Diverse manifestations of quasi-idempotence are exemplified in the synthetic and experimental data presented in the following sections.

\section{SIMULATIONS}

\section{A. Synthetic networks}

The effect of squaring on a graph depends in a non-trivial manner on how its structure eventually leads to an idempotent matrix. Initial insight was gained by considering exemplary binary graphs in preliminary calculations (details not shown). While Erdös-Rényi networks ${ }^{18}$ are not idempotent (i.e., $\iota(1) \approx t(\infty) \approx 0$ ), other random networks can even show "deep" quasi-idempotence when they possess sufficient node degree heterogeneity, as exemplified below for the more general case of weighted graphs. Fully modular networks wherein all communities have equal size (and thus all nodes have equal degree) are perfectly idempotent (i.e., $\iota(1)=\imath(\infty)=1)$; however, when community size is heterogeneous the smaller communities are gradually dissolved, and when separation between modules is incomplete the modular structure is eventually replaced by one generally not resembling the initial network. Arbitrary modular networks thus usually show a "shallow" form of quasiidempotence wherein $t(1)>0$ but $t(\infty) \approx 0$, and a similar scenario is verified for small-world (WattsStrogatz) networks ${ }^{19}$, and for mapped fractal (Sporns) networks ${ }^{6}$ which embody a hierarchy of subnets. The situation for scale-free (Barabási-Albert) networks ${ }^{20}$ is somewhat more complex with $\iota(1) \approx \imath(\infty)$ peaking at $\approx 0.4$ for $m_{0} \approx N / 2$, where $N$ is network size; while these networks are closer to "deep" quasi-idempotence, the peak is not related to scale-freeness but more trivially arises because 
for such setting the network is evenly partitioned into high- and low-degree nodes. Importantly, binary graphs represent a limit scenario for applying the notion of quasi-idempotence as intended here because i) correlation between the elements of the initial and final matrices collapses to a test for difference between two groups (zero or one in the initial matrix), and ii) in complete weighted graphs, the distribution of edge strengths within and between nodes has a strong influence. Hence, binary graphs will not be considered in greater detail and we shall instead restrict attention to complete weighted graphs, which represent the usual starting scenario for structural connection and synchronization matrices derived from experimental neuroscientific data.

Given a complete weighted graph, the Perron-Frobenius theorem implies that the largest eigenvalue of the corresponding matrix is unique and positive, and all elements of the associated eigenvector are positive. Upon squaring the matrix, its column (row) vectors are drawn closer to the direction of the principal eigenvector, yielding a matrix that has a "striped" structure. ${ }^{21}$ After sufficient iterations of squaring, the column (row) vectors are nearly-perfectly aligned with the principal eigenvector, and all other eigenvalues are near-zero, i.e. within numeric precision the matrix is singular and has rank one. Thus repeated squaring effectively corresponds to a "data reduction" operation and the route to idempotence under repeated squaring depends on how these vectors relate to the principal eigenvector in the initial and intermediate matrices. Furthermore, the column (row) sums in the initial and squared matrices are approximately linearly correlated: iterative squaring effectively "locks in" differences in nodal strengths, however small relative to the edge strength distribution these may be, and amplifies them eventually conferring a "striped" structure to the matrix. ${ }^{14-16}$

Based on the above, we hypothesized that in complete weighted graphs the degree of quasiidempotence is related to the distribution of edge strengths, so that quasi-idempotence is enhanced when variance of the edge strengths is determined primarily by heterogeneity of the nodal strengths (i.e., summed or averaged strengths of the edges attached to given nodes), which physically corresponds to a high level of "collectivity" in the dynamics of the system ${ }^{1}$ and visually confers the initial matrix a "striped" structure. To verify this, synthetic networks were generated using the following empirical approach. Let $\varphi_{\mu, \sigma^{2}}(x)$ be a normal probability density function having mean $\mu$ and standard deviation $\sigma$, and without loss of generality let $\mu=0.5$ and $\sigma=0.01$. Let $\varphi_{\mu, \hat{\sigma}^{2}}(x)$ be an array of $N=100$ probability density functions, each consisting of a normal distribution with mean $\mu_{i}$ and standard deviation $\hat{\sigma}$, where $\mu_{i}$ is drawn according to $\varphi_{\mu, \sigma^{2}}(x)$ and $\hat{\sigma}=\eta \sigma$. One can construct a graph $G$ drawing each edge strength $g_{i, j}=g_{j, i}$ according to the "combined" probability density function yielded by

$$
\hat{\varphi}_{i, j}(x)=\sqrt{\varphi_{\mu_{i}, \hat{\sigma}^{2}}(x) \varphi_{\mu_{j}, \hat{\sigma}^{2}}(x)} / \int \sqrt{\varphi_{\mu_{i}, \hat{\sigma}^{2}}(x) \varphi_{\mu_{j}, \hat{\sigma}^{2}}(x)} ;
$$

practically, this operation can be performed numerically by calculating each cumulative distribution function $\hat{\Phi}_{i, j}(x)$ then inverting it. While this process does not realize nodal and edge strength distributions having standard deviation $\sigma$ and $\hat{\sigma}$, it nevertheless offers a simple approach to generate networks wherein the variance of edge strengths unrelated to the heterogeneity of nodal strengths can be straightforwardly controlled by means of parameter $\eta$. For $\eta<<1$, the fraction of edge strength variance unrelated to the nodal strength heterogeneity was very small: the initial matrix thus had a 
characteristic "striped" structure (Fig. 2a), which was preserved by squaring and the degree of quasiidempotence was accordingly very high $(\imath(1) \approx \imath(\infty) \approx 1)$. For $\eta=1$, an intermediate situation was realized, wherein nodal strength heterogeneity was less evident due to greater unrelated edge strength variance: the initial matrix had a weaker "striped" structure (Fig. 2b), and the degree of quasiidempotence was lower because repeated squaring enhanced the nodal strength heterogeneity and attenuated the unrelated edge strength variance, hence the resulting matrix was only moderately correlated with the initial one $(\iota(1) \approx \imath(\infty) \approx 0.5)$. Thus, for both $\eta<<1$ and $\eta=1$, the situation was one of "deep" quasi-idempotence, however with different intensity. On the other hand, for $\eta>>1$, nodal strength heterogeneity was no longer visually appreciable (Fig. 2c) and repeated squaring lead to a pattern devoid of visible element-wise relationship to the initial matrix; similarly to the situation for Erdös-Rényi networks ${ }^{18}$, the matrix was not idempotent $(l(1) \approx l(\infty) \approx 0)$. As confirmed by numerical simulations performed with 100 values of $\eta \log$-spaced between 0.1 and 10 , and 100 networks per setting, $t(1)$ and $t(\infty)$ decreased in unison from $\approx 1$ to $\approx 0$ as $\eta$ was elevated (Fig. $2 \mathrm{~d}$ and Fig. 2e).

The roles of $\sigma$ and $\hat{\sigma}$ in generating these matrices evidently recall the canonical formulation of oneway analysis of variance (ANOVA), wherein the data matrix corresponds to the matrix representing the graph, levels of the factor (groups) correspond to nodes and observations (cases) correspond to edge strengths. ${ }^{22}$ Across these simulations, near-perfect linear correlation was indeed observed between $r_{\mathrm{ANV}}=\sqrt{1-S S E / S S T}$ from such ANOVA and $\imath(\infty)$, with $\imath(\infty) \approx \sqrt{2} r_{\mathrm{ANV}}(r>0.99, m=1.42$, Fig. $2 \mathrm{f}$ ); accordingly, for the matrices obtained by infinite squaring iterations $r_{\mathrm{ANV}} \approx 1 / \sqrt{2}$, and the limit $r_{\mathrm{ANV}}<1 / \sqrt{2}$ holds due to matrix symmetry. While here $t(1) \approx l(\infty)$, the experimental examples presented in the next sections clarify that $r_{\mathrm{ANV}}$ is preferentially related to $t(\infty)$, and may be decoupled from $t(1)$. Throughout the remainder of the study, with the expression "nodal strength heterogeneity" we shall refer to the value of $r_{\mathrm{ANV}}$.

In sum, these results establish a link between self-similarity of edges and triangles in a weighted graph, matrix quasi-idempotence and level of nodal strength heterogeneity as represented by analysis of variance over all edges. Graphs wherein the edge strength variance is strongly related to the heterogeneity of nodal strengths, or equivalently wherein the strengths of the edges attached to each node (matrix row and column vectors) are strongly correlated with the principal eigenvector, or equivalently have non-principal eigenvalues close to zero, possess "deep" quasi-idempotence and are self-similar in the present sense. On the other hand, as demonstrated by the experimental data discussed in the next sections, some networks exhibit "shallow" quasi-idempotence, which also reveals structure formation but is less closely related to nodal strength heterogeneity and eigenvalue spectrum.

\section{B. Kuramoto oscillators}

Since the above networks represent an unphysical scenario, we next considered the paradigmatic case of mean-field (globally-coupled) Kuramoto (phase) oscillators. The collective dynamics of Kuramoto networks under incomplete synchronization support the emergence of complex patterns, and Kuramoto networks have been taken as basis to realize models of oscillatory dynamics in neural ensembles and brain networks, replicating, despite their elementary nature, diverse experimental 
observations including critical phenomena. ${ }^{23-26}$ Namely, we studied a version of the stochastic Kuramoto model, wherein phases $\theta_{i}$ are given by

$\theta_{i}(t)=\omega_{i}+\frac{\lambda}{N} \sum_{j=1}^{N} \sin \left(\theta_{j}(t)-\theta_{i}(t)\right)+\varsigma_{i}(t)$

for $i=1 \ldots N$, where the number of oscillators (network size) $N=100, \omega_{i}$ are the natural frequencies, $\lambda$ denotes coupling strength and $\varsigma_{i}(t)$ denotes white noise drawn from a zero-mean normal distribution having standard deviation $\xi$. We solved the ODE system $40 \times 40$ times assuming different $\lambda$ and $\xi$ values in the range $[0,1]$, set $\omega_{i}$ uniformly to unity and drew $\theta_{i}(0)$ randomly from a homogeneous distribution in $[-\pi, \pi]$. The resulting systems were integrated in 0.001 steps up to $t=1,000$ using the Euler-Maruyama method. ${ }^{27}$ The time-series were decimated to 0.01 steps and the first $10 \%$ was rejected to allow initial transient stabilization. For each combination of $\lambda$ and $\xi, 8$ runs were performed and the final scalar indices were averaged.

Phase synchronization between oscillators is given by the synchronization index

$r_{i, j}=\left|\left\langle e^{i\left[\theta_{i}(t)-\theta_{j}(t)\right]}\right\rangle_{t}\right|$,

according to which average synchronization across the network $\left\langle r_{i, j}\right\rangle$ delineated regions of complete synchronization (for high $\lambda$, low $\xi$ ), incomplete synchronization (for a "band" of intermediate $\lambda$ and $\xi$ ) and complete desynchronization (for low $\lambda$, high $\xi$; Fig. 3a). In the region of incomplete synchronization, the standard deviation of the nodal strengths $\sigma\left\langle r_{i}\right\rangle_{j}$ was maximised (Fig. 3b), and ANOVA indicated that the nodal strength heterogeneity was correspondingly also maximised $\left(r_{\mathrm{ANV}} \approx 1 / \sqrt{2}\right.$; Fig. $3 \mathrm{c}$ ). As previously observed for synthetic networks, also in this case $\imath(1) \approx \imath(\infty)$ (Fig. $3 \mathrm{~d}$ and Fig. 3e), indicating that the synchronization patterns generated by this system yielded different levels of "deep" quasi-idempotence; accordingly, there was very high linear correlation to the ANOVA $r_{\mathrm{ANV}}$-value, with $\imath(\infty) \approx \sqrt{2} r_{\mathrm{ANV}}(r>0.99, m=1.42)$. The underlying synchronization matrices clearly demonstrated the formation of patterns featuring diverse levels of nodal strength heterogeneity in the region of incomplete synchronization, and that repeated squaring enhanced these patterns yielding a "striped" structure (Fig. $3 \mathrm{f}$ and Fig. 3g). While a comprehensive evaluation of the Kuramoto model in this context is beyond the scope of the present work, it was noted that analogous results are also obtained with non-uniform angular frequencies, i.e. drawing $\omega_{i}$ from $[0,1]$ (data not shown).

In the region of incomplete synchronization, as the coupling strength was elevated (or noise was reduced) the collective dynamics of the oscillators became increasingly coherent, eventually converging to a single oscillation mode when complete synchronization was attained. As globallycoherent activity gradually ensued, quasi-idempotence emerged because the level of synchronization attained between a given pair of nodes tracked more and more closely how these "co-participated" in such activity, as represented by their nodal strengths, and accordingly the column (row) vectors of the synchronization matrix became aligned with the principal eigenvector. Such relationship remained 
valid even for the small residual fluctuations observed in presence of strong coupling and low noise. These observations bridge the results from synthetic networks with the experimental data discussed below, providing initial evidence that "deep" quasi-idempotence is a relevant feature of synchronization patterns that can emerge in non-linear dynamical systems, and that it can be viewed as measure of network "collectivity".

\section{EXPERIMENTAL DATA}

\section{A. Electronic chaotic oscillators}

We next considered experimental data acquired in a "micro-scale" setting, wherein each node corresponded to an individual oscillator. For this purpose, we reanalyzed publicly-available timeseries data from a ring network comprising 32 non-linear electronic oscillators having free phase and amplitude, and implemented in field-programmable analog array (FPAA) circuits. This system generates emergent patterns that feature cluster synchronization and diverse forms of remote synchronization; these synchronization patterns consequently have small-world features despite the underlying ring structure, and are reminiscent of aspects of brain functional connectivity. ${ }^{28}$ Collective dynamics were primarily determined by three oscillator parameters, namely two gains $\left(G_{6}, G_{7}\right)$ and an integration constant $\left(K_{1}\right)$, according to the circuit described in detail in Ref. (28). The parameters $G_{6}$ and $G_{7}$ controlled, respectively, the internal loop gain of each oscillator and its coupling with (input from) the preceding one in the ring. As detailed in the original study, for suitable values of $K_{1}$ a "chaotic band" was observed as a function of the two gains, and within it a variety of complex synchronization patterns were formed. Here, we only considered the dataset acquired for $K_{1}=0.11 \mu \mathrm{s}^{-1}$ and restricted network analyses to inside the "chaotic band", defined empirically based on the average correlation dimension of the signals, with $\left\langle D_{2}\right\rangle>1.5$ (Fig. 4a). As detailed in the original study,

synchronization was measured as the maximum cross-correlation coefficient of oscillation amplitude fluctuations $\max \left[C_{i, j}(\tau)\right]$.

While $G_{6}$ and $G_{7}$ were elevated, fine-grained structure appeared within the "chaotic band", visible as variability in the average correlation dimension $\left\langle D_{2}\right\rangle$ and amplitude synchronization $\left\langle\max \left[C_{i, j}(\tau)\right]\right\rangle$

(Fig. 4a and Fig. 4b). Considerable fluctuation of the ANOVA $r_{\mathrm{ANV}}$-value was correspondingly observed, indicating that small changes in $G_{6}$ and $G_{7}$ lead to the generation of synchronization patterns expressing diverse levels of nodal strength heterogeneity (Fig. 4c). In contrast to the synthetic networks and Kuramoto oscillators considered above, here a clear dissociation appeared between $t(1)$ and $\imath(\infty)$, which were only moderately correlated $(r=0.42)$; consideration of the respective distributions with respect to $G_{6}$ and $G_{7}$ revealed that while for low $G_{6}$ and high $G_{7}$ synchronization patterns pervasively exhibited "shallow" quasi-idempotence (Fig. 4d), "deep" quasi-idempotence only emerged for narrower control parameter regions (Fig. 4e). Linear correlation between the ANOVA $r_{\mathrm{ANV}}$-value and $\iota(\infty)$ was again near-perfect with $\iota(\infty) \approx \sqrt{2} r_{\mathrm{ANV}}(r>0.99, m=1.58)$, however correlation with $\imath(1)$ was much weaker $(r=0.37)$.

Consideration of the underlying synchronization matrices demonstrated that "deep" quasiidempotence was primarily supported by remote synchronization appearing in the form of heightened synchronization between distant ring segments, which lead to markedly heterogeneous nodal 
strengths; notably, the communities formed via cluster synchronization in this system (visible along the diagonal in the initial synchronization matrices) were not preserved during repeated squaring (Fig. $4 \mathrm{f}$ and Fig. 4g). Contrariwise, "shallow" quasi-idempotence was observed for synchronization matrices exhibiting primarily another form of remote synchronization which ensued between node pairs having approximately fixed non-unitary distance (appearing as "diagonal lines" on the initial matrix, Fig. 4h), or exhibiting weaker pattern formation in the form of community substructure overlaid to a synchronization gradient decaying with node separation (Fig. 4i): although largely preserved in the first iteration, both patterns were eventually dissipated by repeated matrix squaring.

These results are consistent with those from mean-field Kuramoto oscillators, but additionally emphasize the different sensitivities of $t(1)$ and $t(\infty)$ thanks to the richer repertoire of synchronization patterns available to this system: while both measures were sensitive to spontaneous pattern formation, high $t(\infty)$ required large nodal strength heterogeneity that in this case was only conferred by remotely synchronized clusters ("deep" quasi-idempotence), whereas $\imath$ (1) was also elevated in presence of other less stable substructures ("shallow" quasi-idempotence). While in Kuramoto networks the emergence of "deep" quasi-idempotence straightforwardly reflected co-participation in increasingly coherent global activity, in this scenario the situation was more complex because the system could generate a repertoire of qualitatively-different synchronization patterns. ${ }^{28}$

\section{B. Neural cultures on multi-electrode arrays}

Subsequently, we considered a biological "meso-scale" scenario, wherein each network node ensumed activity from a population of contiguous oscillators. For this purpose, we reanalyzed publicly-available spike series data of cortical neurons harvested from rat embryos, dissociated, then plated on culture wells containing multi-electrode arrays (MEA). While the initial connectivity is destroyed, the plated neurons spontaneously grow new axonal connections over a scale of few millimetres, eventually leading to generation of a rich repertoire of bursting patterns, critical dynamics and complex network topology. ${ }^{29-31}$ We drew data from so-called "dense" cultures, wherein $\approx 50,000$ cells were concentrated in a droplet and plated on area having diameter $\approx 5 \mathrm{~mm}$, underneath which an array of $8 \times 8$ recording electrodes with pitch $200 \mu \mathrm{m}$ was instanced. Spontaneous spiking activity was recorded daily for $30 \mathrm{~min}$ from 10 until 40 days in-vitro (DIV), and due to inherent biological variability the cultures were substantially heterogeneous in activity intensity and features. ${ }^{29}$ Because of limited data after 35 DIV, we restricted correlation analyses up to this culture age.

After performing spike detection, which yielded a point-process activity representation, bursts were identified separately for each electrode based on temporal clustering (minimum inter-spike interval $\approx 0.1 \mathrm{~s}$, adaptively set). ${ }^{32}$ Subsequently, we segmented network bursts intended as time-intervals of simultaneous bursting involving $\geq 5$ electrodes; further analyses only considered activity within these intervals, as it is during them that synchronization is preferentially expressed over the background of stochastic firing. Synchronization matrices were thereafter obtained by computing the coincidence index (CI), which represents the cross-correlation integral within a \pm 1 ms conduction delay with respect to the total. ${ }^{31}$ The number of active electrodes depended on culture age and individual features, and network size varied between 6-58 nodes. 
Reflecting the expected spontaneous growth of new connections (i.e., synapses, axons, dendritic trees) among the initially-dissociated neurons, the number of active electrodes (network size $N$ ) raised steadily (rank-order $r=0.40$; Fig. 5a), and the average synchronization among them also increased $\left(r=0.64\right.$; Fig. $5 \mathrm{~b}$ ). Notably, nodal strength heterogeneity as measured by the ANOVA $r_{\mathrm{ANV}}$-value also gradually raised $(r=0.58)$, featuring a steeper gradient between 10-20 DIV and a shallower one afterwards (Fig. 5c). Correspondingly, $\imath(1)$ and $t(\infty)$ increased in unison $(r=0.63$ and $r=0.58$ respectively; Fig. 5d and Fig. 5e); here, they were highly linearly related $(r=0.93)$, and as observed previously, $t(\infty)$ was near-perfectly correlated to the ANOVA $r_{\mathrm{ANV}}$-value with $\imath(\infty) \approx \sqrt{2} r_{\mathrm{ANV}}(r>0.99$; $m=1.41)$.

Consideration of the underlying synchronization matrices revealed a clear progression in structure formation between immature and fully-developed cultures. Initially, less than two weeks after plating, the distribution of edge strengths appeared random, and yielded low $\imath(1)$ and $t(\infty)$ (Fig. 5f). Already after three weeks, stable substructures pervasively appeared in the form both of individual highstrength nodes, and of large communities conferring higher nodal strength to their members, yielding intermediate and concordant values of $t(1)$ and $t(\infty)$ (Fig. 5g). From that culture age onwards, the majority of cultures further evolved towards markedly quasi-idempotent topology, driven by emergence of groups of high-strength nodes, which also preferentially synchronized between themselves; values of $\imath(1)$ and $\imath(\infty)$ were accordingly high (Fig. 5h and Fig. 5i).

As $t(1) \approx t(\infty)$, these results demonstrate that self-organized structure formation in biological neural networks leads to the emergence of "deep" quasi-idempotence, which in this case reflected the increasing collective participation in coherent activity ensuing as network-wide bursts, recalling the situation for Kuramoto oscillators. There was striking correlation with culture development stage (age), which was evident particularly in early weeks when new connections are developed rapidly; compared to previous observations in this area predicated on other network topology measures, these results suggest that quasi-idempotence has remarkable sensitivity to the emergent organization exhibited by these networks. ${ }^{33}$

\section{Brain structure and function in human and mouse}

We finally considered "macro-scale" brain architecture in terms of both structural and functional connectivity, accessed via magnetic resonance imaging (MRI) techniques respectively probing axonal bundle anatomy and hemodynamic activity, and via meta-analysis of an activation data repository. In the resulting networks, each node effectively corresponded to the ensemble average over a cortical parcel which, depending on local cytoarchitecture and parcellation granularity, could harbour $\approx 10^{6}$ $10^{8}$ neurons. Structural and functional brain connectivity is concomitantly characterized by multiple topological features, which are deemed to have emerged under evolutionary pressure as means to enhance processing capacity relative to size and metabolic cost; these include small-world, scale-free organization, hierarchical modular organization and presence of a "rich-club" of hub nodes that are both heavily interconnected to the rest of the cortex, and preferentially interconnected between themselves. ${ }^{8-10,34-38}$

To evaluate the degree of quasi-idempotence of macroscopic brain networks, we first considered axonal connectivity, averaged over 5 healthy, young participants who were studied by means of 
diffusion-spectrum imaging (DSI), an MRI technique which relies upon water self-diffusion anisotropy to probe white matter micro-structure. Publicly-available data were drawn from a previous study wherein DSI was performed sampling the $Q$-space over 129 points in $2 \times 2 \times 3 \mathrm{~mm}$ voxels, followed by reconstruction of the orientational probability density functions and tractography via a deterministic streamline algorithm, which yielded "fibres" representing an approximation of the underlying anatomy. For this and the other data sources referred to below, ethics statements are contained in the original publications. To construct the connectivity matrix, the cortex was parcellated in 998 regions, pair-wise connection density was determined based on fibre count and length, then the resulting values were logarithm-transformed and rescaled to $[0,1] .^{35}$ The resulting network was sparse, having completeness $8 \%$, and featured a prominent distinction between intra- and inter-hemispheric connections. Squaring the matrix once enhanced the connectivity of high-strength nodes, and yielded a network highly correlated to the initial one, having $\imath(1)=0.78$; further iterations consolidated the predominance of such nodes and dissolved the unrelated substructures, eventually yielding weak correlation with the initial matrix, having $\imath(\infty)=0.20$ (Fig. 6a), indicating that the network possessed "shallow" quasi-idempotence. Anatomical visualization of nodal strength distribution highlighted that it was highest for contiguous parcels clearly delineating the boundaries of the precuneus and posterior cingulate cortex, which knowingly represent the principal hub in the "rich-club" of brain connectivity, and squaring effectively had the effect of "focusing" connectivity onto these areas (Fig. 7a). ${ }^{39,40}$ Even though the situation was one of "shallow" quasi-idempotence, both indices were significantly higher $\left(p<10^{-6}\right)$ than for null networks obtained via random edge permutation $(n=100)$, which preserved edge strength but not nodal strength distribution, and yielded $t(1)=0.000 \pm 0.003$ and $t(\infty)=0.045 \pm 0.001$

(mean \pm standard deviation). Here, as a consequence of matrix sparsity, $\sqrt{2} r_{A N V}<\imath(\infty)$ with $\sqrt{2} r_{\mathrm{ANV}}=0.13$.

We then drew from another study the average functional connectivity measured over 24 healthy, young participants, in whom blood oxygen level-dependent time series were recorded during idle wakefulness, for 150 time-points spaced by $2 \mathrm{~s}$, voxel size $3.5 \times 3.5 \times 3 \mathrm{~mm}$ (resting-state functional MRI, rs-fMRI). After suitable filtering to attenuate variance due to non-neural physiological activity, low-frequency fluctuations $(<0.1 \mathrm{~Hz})$ in these signals knowingly represent spontaneous brain activity that is coherent across cortical regions, separable in a set of independent components and simultaneously shows complex network architecture. ${ }^{25,41}$ To construct the connectivity matrix, the cortex was parcellated in 638 regions, pair-wise temporal correlations were calculated and Fisher transformed, discarding negative correlations as their interpretation is controversial. ${ }^{37}$ The resulting network was near-complete at $99.8 \%$ and featured a well-evident subdivision in default-mode, visual, fronto-parietal and central modules, in line with previous reports. Also for this network, moderate nodal strength heterogeneity was present and enhanced by squaring the matrix once, yielding $\imath(1)=0.72$; however, compared to structural connectivity, this network had more stable structure and repeated squaring retained moderate correlation to the initial matrix, with $\imath(\infty)=0.57$ (Fig. 6b), indicating an "intermediate" degree of quasi-idempotence, also evident as close preservation of the nodal strength distributions before and after squaring (Fig. 7b). Both indices were again significantly higher $\left(p<10^{-6}\right)$ than for null networks obtained via random edge permutation, which yielded $\imath(1)=-0.000 \pm 0.003$ and $\iota(\infty)=0.056 \pm 0.001$ (mean \pm standard deviation); in this case $t(\infty) \approx \sqrt{2} r_{\mathrm{ANV}}$, with $\sqrt{2} r_{\mathrm{ANV}}=0.55$. 
We furthermore drew from the same study statistical data on co-activation, determined between all region pairs by meta-analysis of $>1,600$ studies available in the BrainMap database ${ }^{42}$, performed using fMRI or positron-emission tomography (PET) and contrasting active tasks in diverse domains including language, memory, other cognitive functions, emotion, sensory stimulation and motor performance. The same cortical parcellation in 638 regions was assumed, but in this case matrix entries consisted of Jaccard similarity scores. Effectively, this provided a measure of synchronization which was predicated on variance among studies and tasks instead of temporal variance of spontaneous activity during idleness, and therefore potentially more ecologically valid. As detailed in the original work, the resulting network had significant correspondence to spontaneous activity synchronization measured during idleness and featured a similar modular organization. ${ }^{37}$ Although more sparsely connected, having completeness $83.9 \%$, also this network demonstrated moderate nodal strength heterogeneity, which was enhanced by squaring the matrix once, yielding $\imath(1)=0.62$; it had marginally less stable structure than synchronization of spontaneous activity during idleness, and repeated squaring lead to weaker correlation with the initial matrix, with $\imath(\infty)=0.43$ (Fig. 6c). Highstrength nodes were located primarily in superior frontal, superior parietal regions and lateral frontal regions, in line with the distribution of central "rich club" hubs identified previously, and their distribution was closely preserved by squaring (Fig. 7c) ${ }^{37}$ Both indices were significantly higher $\left(p<10^{-6}\right)$ than for null networks obtained via random edge permutation, which yielded $\iota(1)=-0.000 \pm 0.003$ and $\iota(\infty)=0.056 \pm 0.002$ (mean \pm standard deviation); here, $\sqrt{2} r_{\mathrm{ANV}}=0.36$.

Lastly, we considered murine structural connectivity, which reflects a type of brain anatomy that is profoundly different to the human one due to lower gyration, greater centrality of subcortical structures and different architecture of the main pathways. ${ }^{43}$ To this end, we obtained from another public repository the average structural connectivity matrix of 2 wild-type adult C57BL/6 mice. ${ }^{44}$ These were studied ex-vivo with diffusion-tensor imaging (DTI), probing water self-diffusion along 120 directions in $43 \mu \mathrm{m}$ isotropic voxels and subsequently performing probabilistic tractography, generating probability maps starting from each of 296 parcellation regions. To construct the connectivity matrix, for each region pair the relative connectivity estimates were averaged over mice and between directions to yield a symmetric matrix, and the resulting values were logarithmtransformed and rescaled to $[0,1]$. The resulting network had completeness $98.6 \%$, and a prominent modular structure delineating the isocortex, pallidum-diencephalon-midbrain, and hindbrain. There was moderate nodal strength heterogeneity, which was enhanced by squaring the matrix once, with $\imath(1)=0.74$, and relatively stable with respect to further squaring, with $t(\infty)=0.65$ (Fig. 6d), pointing to "deep" quasi-idempotence in apparent contrast with human data. High-strength nodes were located primarily in the pallidum and subpallidum, in keeping with the central role of these structures in murine brain architecture. ${ }^{45}$ Both indices were significantly higher $\left(p<10^{-6}\right)$ than for null networks obtained via random edge permutation, which yielded $\imath(1)=0.000 \pm 0.007$ and $\imath(\infty)=0.083 \pm 0.003$ (mean \pm standard deviation); here, $\sqrt{2} r_{\mathrm{ANV}}=0.66$.

We additionally evaluated the quasi-idempotence of structural and functional connectivity in the context of publicly-available data acquired from a large cohort of healthy human participants representative of the entire lifespan (NKI-Rockland sample, obtained via the UCLA multimodal connectivity database). ${ }^{46,47}$ Connectivity matrices according to a parcellation in 188 regions were 
obtained based, respectively, on DTI and rs-fMRI measurements, applying methods detailed elsewhere; in these analyses, we did not logarithm-transform the DTI-derived connection weights and we only considered rs-fMRI data without global-signal regression. For DTI ( $n=196$, age 35.0 \pm 20.0 years), we obtained $\iota(1)=0.79 \pm 0.01$ (mean \pm standard deviation) and $\iota(\infty)=0.42 \pm 0.04$, whereas for rsfMRI ( $n=146$, age 35.4 \pm 20.1 years), we obtained $\imath(1)=0.81 \pm 0.03$ and $t(\infty)=0.59 \pm 0.11$. These measurements demonstrated that quasi-idempotence is highly consistent across individuals and age ranges. Notably, there was a moderate inverse correlation between $\imath(1)$ derived from DTI and age (rank-order $r=-0.24, p<0.001$ ); this effect, however, should be interpreted cautiously as it was quantitatively very small. No such correlations was observed for $\imath(\infty)(p=0.6)$, and for rs-fMRI measures there was only a marginal, unconvincing correlation $(p=0.03)$. These results suggest that age-related degeneration could weaken the topological features that underpin quasi-idempotence, and do so primarily affecting structural connectivity, as observed elsewhere for other network measures. 48,49

Taken together, these findings confirm that significant self-similarity between edges and paths is to some extent present even in the macroscopic organization of brain networks, and demonstrate quasiidempotence in the context of networks substantially larger than those considered in the previous scenarios. Quasi-idempotence was verified for structural networks in both human and mouse brains despite profound anatomical differences, and for synchronization as well as co-activation networks in the human brain. For structural connectivity, it is plausible that the different degree of idempotence observed between the species was due to both biological factors (white matter architecture, brain size, gyration) and methodological aspects (tracking algorithm, resolution, thresholding/sparsity, in-vivo vs. in-vitro). For functional connectivity, differences between datasets may be down to confounding effects of parcellation and signal filtering differences. Predicated on the clearly different effect of squaring on nodal strength topography visible in Fig. 7, we speculate that at this level hardwired structural (axonal) connectivity may preferentially express "shallow" quasi-idempotence, whereas "deep" quasi-idempotence may become apparent only for emergent synchronization patterns (functional connectivity and co-activation). These results additionally indicate that measurements were reproducible and potentially sensitive to age-related degeneration.

\section{DISCUSSION}

This study investigated network self-similarity by means of a novel approach that considers edges and paths, rather than nested hierarchies of nodes and modules as already done extensively in the existing literature. ${ }^{50,51}$ It was demonstrated that for the case of triangles, namely the shortest possible nonunitary-length paths, rescaling corresponds to matrix squaring, and consequently that network selfsimilarity corresponds to matrix quasi-idempotence, intended as a "relaxed" version of the canonical definition of idempotence based on linear correlation of off-diagonal elements. Two scalar indices that encapsulate the "degree" of quasi-idempotence were proposed, alongside a qualitative distinction between "shallow" and "deep" quasi-idempotence, predicated on observations that certain emergent networks possess local edge substructures that are stable over few squaring iterations but are eventually dissolved or distorted, while others feature a more globally-stable topology that is close to the one that iterative squaring eventually converges to. It was shown that quasi-idempotence does not trivially reflect already well-known network properties such as scale-freeness and small-worldness, and that even though multiple elementary topologies possess "shallow" quasi-idempotence, "deep" 
quasi-idempotence is related to nodal strength heterogeneity in a clear manner, such that the most stable networks are those wherein edge variance is predominantly determined by the nodal strengths, a property which maps closely to the definition of statistical difference as embodied in analysis of variance (ANOVA).

To the authors' knowledge, the notion of matrix idempotence had thus far been applied in the context of graph theory only sparingly, with the notable exception of the MCL algorithm, where expansion and inflation steps, corresponding to matrix and Hadamard products, are alternated to obtain optimal graph partitioning. In this context, it is established that squaring a matrix (expansion) corresponds to increasing the path lengths that are considered, in other words, looking at a coarser scale. It was shown that matrix idempotence is related to the presence of stable substructures rich in triangular motifs. ${ }^{52}$ While the expansion step is in common between our approach and the MCL, our take is different, in that no inflation is performed and the purpose is to measure the degree of quasiidempotence and not to partition graphs. Furthermore, our approach to self-similarity is reminiscent of the matching index, an auxiliary matrix used in the context of network clustering wherein each element quantifies the overlap in neighbours between each pair of nodes ${ }^{53,54} \mathrm{~A}$ similar notion has also been proposed as a means of detecting missing links. ${ }^{55}$ However, while the matching index is a bounded measure of overlap, in our case the matrix entries after squaring represent the sum of paths between node pairs across iterations, hence, two nodes can have a high matching but nevertheless be weakly connected to the rest of the network overall, and vice versa. Future studies should consider the relationship between quasi-idempotence as intended in this context, and the aspects of network symmetry reflected by the Laplacian spectra as highlighted in recent work. ${ }^{56}$

As summarized in Table 1, convergent observation of quasi-idempotence in profoundly different dynamical systems, namely Kuramoto networks, electronic chaotic oscillators, neural cultures and even entire brains motivates querying the significance of this property. One perspective is yielded by considering it as a measure of "collectivity", as clearly exemplified by observations in incompletelysynchronized Kuramoto networks. ${ }^{1}$ If in the lifetime of a given network links (representing structural connections or activity synchronization, depending on the nature of the graph) are formed, strengthened and maintained in a manner that is insensitive to global activity (low collectivity), the variance of edge strengths is largely uncoupled to nodal strength heterogeneity and quasi-idempotence is accordingly low ( $\eta>>1$ in subsection III.A). If on the other hand, links between nodes reflect how intensely these co-participate in a coherent activity mode or in a coherent structure yielding them a certain level of centrality in the network (high collectivity), then the variance of edge strengths is largely determined by nodal strength heterogeneity, and quasi-idempotence is high ( $\eta<<1$ in subsection III.A). This appears to be the case for spontaneously-developing in-vitro neural networks, wherein maturation is associated with increasingly frequent generation of bursts of network-wide coherent activity, and correspondingly higher quasi-idempotence emerging alongside other topological features. ${ }^{30,31}$ As regards to structural connectivity and neural plasticity, quasi-idempotence can be supported by Hebb's rule, according to which neurons that are driven to discharge in synchrony by collective dynamics develop strong direct connection. ${ }^{57}$ Another perspective is yielded by considering that quasi-idempotence represents a scenario wherein the efficiency of information transfer between any two given nodes is coupled, whether transfer takes place directly between them or diffusely via the rest of the network, which could maximise network resilience. ${ }^{58,59}$ "Economic" arguments about network efficiency and resilience should however considered cautiously when 
examining the significance of quasi-idempotence, considering that this property was also clearly observed in simulated and physical oscillator networks, whose features do not reflect an evolutionary response to environmental pressure as may be the case for neural networks in-vivo and in-vitro. ${ }^{9}$ Quasi-idempotence could, nevertheless, represent a form of "minimum energy configuration", towards which collective dynamics are drawn regardless of the nature of the system. ${ }^{60}$

Future theoretical, numerical and experimental investigations are motivated to understand the underlying causes and the consequences of this kind of network self-similarity for collective dynamics, and to better clarify the distinction between and implications of "shallow" and "deep" quasi-idempotence (which herein remained defined qualitatively, without a rigorous, formal criterion), as well as the relationship to other topological properties. In particular, while this study has focused on small, complete graphs, there is a need to determine whether this form of self-similarity is also observed in the very large, sparse topologies that are typical of social, transportation and biological networks which feature well-understood properties such as small-worldness and scale-freeness; element-wise correlation appears poorly suitable for such networks, hence alternative measures of similarity may be required. .,19,20,61 $^{2}$

In this study, only the case $l=1$ was considered, in other words paths were represented exclusively by triangles, leading to the question of what would be observed for longer paths. We posit that even though for small number of iterations $n$ the number of intermediate nodes $l$ may have substantial impact, in $n \rightarrow \infty$ it does not since the ergodic limit is reached regardless of $l$. This assertion requires verification in future work, and more generally there is a need to investigate other potential measures of quasi-idempotence (sensitive, for example, to non-linear correlation), and the influence of network size and edge strength distribution (including sparsity). Further, the empirical correspondence between correlation of matrix elements after infinitely-repeated squaring $(l(\infty))$ and canonical analysis of variance $\left(r_{\mathrm{ANV}}\right)$ is particularity striking given that the latter is yielded by a close-form expression, and requires deeper investigation, also given that the correspondence was not universally valid as typified by results in sparse networks and by the pathological case of perfectly modular networks with evenly-sized communities, which have $\imath(\infty)=1$ but $r_{\mathrm{ANV}}=0$. It will furthermore be necessary to confirm the present results in the context of a careful evaluation of the effects of connectivity matrix calculation methods on quasi-idempotence, in particular in the context of macroscopic brain networks by determining the effects of data filtering, cortical parcellation density, weight scaling and thresholding choices. ${ }^{62}$

Further work is also necessary to elucidate the relevance of quasi-idempotence to neural networks probed at finer scale via synaptic maps and single-cell recordings, and other graphs such as gene regulatory networks and protein-protein interactions. The present observations also point to quasiidempotence as a possible basis for novel network integrity biomarkers, particularly in the context of neuropsychiatric disease connectomics: since strong expression of quasi-idempotence requires tight coupling between edge and nodal strengths, effectively indexing local and global aspects of brain connectivity, alterations affecting either level should be readily detectable. ${ }^{63}$ For instance, lower quasi-idempotence is expected in autism due to weaker influence of global activity on regional plasticity, and in Alzheimer's disease, wherein synaptic loss preferentially targets the highest-node degree hubs, gradually dissolving the nodal heterogeneity which drives edge strengths in networks 
that possess strong quasi-idempotence. ${ }^{64,65}$ Re-analysis of existing datasets and computational investigations in this direction appear motivated.

\section{Acknowledgements}

LM produced the initial version of this study while at the University of Trento (Italy) and generously funded by Scienze Mente-Cervello S.c.a.r.l. (Rovereto, Italy), and subsequently developed it further while employed at the Institute of Nuclear Physics - Polish Academy of Science (Kraków, Poland). The authors thank Stanisław Drożdż and Vladimir Vlasov for insightful advice respectively on the relationship between eigenvectors and repeated squaring, and on Kuramoto networks. The authors are grateful to i) Daniel A Wagenaar, Steve M Potter and colleagues for providing neural cultures data, their work was supported from NINDS grants NS38628 and NS44134, NIBIB grant EB00786, by the NSF Center for Behavioral Neuroscience and Whitaker Foundation; ii) Patric Hagmann and colleagues for providing the human DTI data, their work was supported by grants for interdisciplinary biomedical research to the University of Lausanne, the Department of Radiology of University Hospital Center in Lausanne (CHUV), the Center for Biomedical Imaging (CIBM) of the Geneva Lausanne Universities and the Ecole Polytechnique Fédérale de Lausanne (EPFL), as well as grants from the foundations Leenaards and Louis-Jeantet and Mr Yves Paternot, JS McDonnell Foundation, NIMH grant 1R01-MH64-44; iii) David Crossley and colleagues for providing human functional connectivity and co-activation data, their work was supported by Wellcome Trust, UK NIHR and AFOSR grant FA9550-12-1-0102, and based on the BrainMap project, funded by NIMH award R01MH074457 to Peter T. Fox and Angela R. Laird; iv) Evan Calabrese and colleagues regarding mouse DTI data, which were provided by the Duke Center for In Vivo Microscopy supported by the NIH/NIBIB (P41 EB015897) with additional support from (1S10OD010683-1); v) Kate Brody Nooner and colleagues regarding NKI-RS data, their work was primarily funded by grants NIMH BRAINS R01MH094639-01 (PI Milham), NIMH R01MH081218, R01MH083246, and R21MH084126, NKI Center for Advanced Brain Imaging (CABI), the Brain Research Foundation (Chicago, IL), and the Stavros Niarchos Foundation. The authors further acknowledge the contribution of the USC Multimodal Connectivity Database, supported by NIH NRSA F31AG035438-01 to Jesse A. Brown and NIH Neuroscience Blueprint HHSN271200800035C via NIDA. Full listing and further particulars in the cited data sources.

\section{References}

${ }^{1}$ J. Kwapień and S. Drożdż, Phys. Rep. 515, 115 (2012)

${ }^{2}$ S. Achard, D.S. Bassett, A. Meyer-Lindenberg, and E. Bullmore, Phys. Rev. E 77, 036104 (2008)

${ }^{3}$ D.R. Chialvo, Nat. Phys. 6, 744 (2010)

${ }^{4}$ P. Expert, R. Lambiotte, D.R. Chialvo, K. Christensen, H.J. Jensen, D.J. Sharp, and F. Turkheimer, J.

R. Soc. Interface 8, 472 (2011)

${ }^{5}$ O. Plenz, O. Niebur, and H.G. Schuster, Criticality in Neural Systems (Wiley, Hoboken NJ, USA, 2014)

${ }^{6}$ O. Sporns, BioSystems 85, 55 (2006)

${ }^{7}$ G. Werner, Front. Physiol. 1, 15 (2010)

${ }^{8}$ M.P. van den Heuvel, R.S. Kahn, J. Goñi, and O. Sporns, Proc. Natl. Acad. Sci. USA 109, 11372

(2012)

${ }^{9}$ E. Bullmore and O. Sporns, Nat. Rev. Neurosci. 13, 336 (2012) 
${ }^{10}$ S.S. Singh, B. Khundrakpam, A.T. Reid, J.D. Lewis, A.C. Evans, R. Ishrat, B.I. Sharma, and R.K. Singh, Sci. Rep. 6, 24926 (2016)

${ }^{11}$ S. Lorthois, F. Cassot, and A. Di Ieva, The Fractal Geometry of the Brain (Springer, New York NY, USA, 2016).

${ }^{12}$ C. Song, S. Havlin, and H.A. Makse, Nature 433, 392 (2005)

${ }^{13}$ B. Peirce, Linear Associative Algebra (Washington, USA, 1870)

${ }^{14}$ K.M. Hoffman and R. Kunze, Linear Algebra (Pearson, New York NY, USA, 1971)

${ }^{15} \mathrm{~K}$. Hoffman, Analysis in Euclidean Space (Dover Editions, Mineola NY, USA, 2007)

${ }^{16}$ G. Akemann, J. Baik, and P. Di Francesco, The Oxford Handbook of Random Matrix Theory (Oxford University Press, Oxford, UK, 2011)

${ }^{17}$ K. Atkinson, An Introduction to Numerical Analysis (Wiley, Hoboken NJ, USA, 1989)

${ }^{18} \mathrm{P}$. Erdős and A. Rényi, On the evolution of random graphs, Publ Math Inst Hung Acad Sci 5, 17 (1960)

${ }^{19}$ D.J. Watts and S.H. Strogatz, Nature 393, 440 (1998)

${ }^{20}$ A.L. Barabási and R. Albert, Science 286, 509 (1999)

${ }^{21}$ K. Delp, Seoul Conference Proceedings 73-78 (Seoul, South Korea, 2014)

${ }^{22}$ R.A. Fisher, Statistical Methods for Research Workers (Macmillan Publishers, London, UK, 1970)

${ }^{23}$ J.A. Acebrón, L.L. Bonilla, C.J. Pérez Vicente, F. Ritort, and R. Spigler, Rev. Mod. Phys. 77, 137 (2005).

${ }^{24}$ M.G. Kitzbichler, M.L. Smith, S.R. Christensen, and E. Bullmore, PLoS Comput Biol 5, e1000314 (2009)

${ }^{25}$ J. Cabral, M.L. Kringelbach, and G. Deco, Prog. Neurobiol. 114, 102 (2014)

${ }^{26}$ F.A. Rodrigues, T. Peron, H. Peng, and J. Kurths, Phys. Rep. 610, 1 (2016)

${ }^{27}$ P.E. Kloeden and E. Platen, Numerical Solution of Stochastic Differential Equations (Springer, Berlin, Germany, 1992)

${ }^{28}$ L. Minati, CHAOS 25, 123107 (2015)

${ }^{29}$ D.A. Wagenaar, J. Pine, and S.M. Potter, BMC Neurosci 7, 1 (2006)

${ }^{30}$ J.H. Downes, M.W. Hammond, D. Xydas, M.C. Spencer, V.M. Becerra, K. Warwick, B.J. Whalley, and S.J. Nasuto, PLoS Comput Biol 8, e1002522 (2012)

${ }^{31}$ P. Massobrio, V. Pasquale, and S. Martinoia, Sci. Rep. 5, 10578 (2015)

${ }^{32}$ D.A. Wagenaar, T.B. DeMarse, and S.M. Potter, Proc 2nd Intl IEEE EMBS Conf on Neural Eng. $518(2005)$

${ }^{33}$ D. Poli, V.P. Pastore, and P. Massobrio, Front Neural Circuits 9, 57 (2015)

${ }^{34}$ O. Sporns, Networks of the Brain (MIT Press, Cambridge MA, USA, 2009).

${ }^{35}$ P. Hagmann, L. Cammoun, X. Gigandet, R. Meuli, C.J. Honey, V.J. Wedeen, and O. Sporns, PLoS Biol 6, e159 (2008)

${ }^{36}$ E. Bullmore and O. Sporns, Nat Rev Neurosci 10, 186 (2009)

${ }^{37}$ N.A. Crossley, A. Mechelli, P.E. Vértes, T.T. Winton-Brown, A.X. Patel, C.E. Ginestet, P.

McGuire, and E.T. Bullmore, Proc. Natl. Acad. Sci. USA 110, 11583 (2013)

${ }^{38}$ S.E. Petersen and O. Sporns, Neuron 88, 207 (2015)

${ }^{39}$ M. Xia, J. Wang, and Y. He, PLoS One 8, e68910 (2013)

${ }^{40}$ A.E. Cavanna and M.R. Trimble, Brain 129, 564 (2006)

${ }^{41}$ C. Rosazza and L. Minati, Neurol. Sci. 32, 773 (2011)

${ }^{42}$ P.T. Fox and J.L. Lancaster, Nat. Rev. Neurosci. 3, 319 (2002)

${ }^{43}$ S.W. Oh, J.A. Harris, L. Ng, B. Winslow, N. Cain, S. Mihalas, Q. Wang, C. Lau, L. Kuan, A.M. Henry, M.T. Mortrud, B. Ouellette, T.N. Nguyen, S.A. Sorensen, C.R. Slaughterbeck, W. Wakeman, 
Y. Li, D. Feng, A. Ho, E. Nicholas, K.E. Hirokawa, P. Bohn, K.M. Joines, H. Peng, M.J. Hawrylycz, J.W. Phillips, J.G. Hohmann, P. Wohnoutka, C.R. Gerfen, C. Koch, A. Bernard, C. Dang, A.R. Jones, and H. Zeng, Nature, 508, 207 (2014)

${ }^{44}$ E. Calabrese, A. Badea, G. Cofer, Y. Qi, and G. Allan Johnson, Cereb Cortex 25, 4628 (2015)

${ }^{45}$ H. Hintiryan, N.N. Foster, I. Bowman, M. Bay, M.Y. Song, L. Gou, S. Yamashita, M.S.

Bienkowski, B. Zingg, M. Zhu, X.W. Yang, J.C. Shih, A.W. Toga, and H.W. Dong, Nat. Neurosci. $19,1100(2016)$

${ }^{46}$ K.B. Nooner, S.J. Colcombe, R.H. Tobe, M. Mennes, M.M. Benedict, A.L. Moreno, L.J Panek, S. Brown, S.T. Zavitz, Q. Li, S. Sikka, D. Gutman, S. Bangaru, R.T. Schlachter, S.M. Kamiel, A.R. Anwar, C.M. Hinz, M.S. Kaplan, A.B. Rachlin, S. Adelsberg, B. Cheung, R. Khanuja, C. Yan, C.C. Craddock, V. Calhoun, W. Courtney, M. King, D. Wood, C.L. Cox, A.M. Kelly, A. Di Martino, E. Petkova, P.T. Reiss, N. Duan, D. Thomsen, B. Biswal, B. Coffey, M.J. Hoptman, D.C. Javitt, N. Pomara, J.J. Sidtis, H.S. Koplewicz, F.X. Castellanos, B.L. Leventhal, and M.P. Milham, Front Neurosci 6, 152 (2012)

${ }^{47}$ J.A. Brown, J.D. Rudie, A. Bandrowski, J.D. Van Horn, and S.Y. Bookheimer, Front Neuroinform 6, 28 (2012)

${ }^{48}$ S. Hirsiger, V. Koppelmans, S. Mérillat, F. Liem, B. Erdeniz, R.D. Seidler, and L. Jäncke, Hum Brain Mapp 37, 855 (2016)

${ }^{49}$ A.M. Fjell, M.H. Sneve, H. Grydeland, A.B. Storsve, and K.B. Walhovd, Cereb Cortex, Epub ahead of print (2016)

${ }^{50} \mathrm{O}$. Sporns, G. Tononi, and R. Kötter, The human connectome: A structural description of the human brain, PLoS Comput Biol 1, e42 (2005)

${ }^{51}$ H.C. Barrett, Proc. Natl. Acad. Sci. USA 109, 10733 (2012)

${ }^{52}$ S. Van Dongen, SIAM J. Matrix. Anal. Appl. 30, 121 (2008)

${ }^{53}$ O. Sporns, C.J. Honey, and R. Kötter, PLoS One 2, e1049 (2007)

${ }^{54}$ G. Zamora-López, C. Zhou, and J. Kurths, Front. Neuroinform. 4 (2010)

${ }^{55}$ D. Liben-Nowell and J. Kleinberg, CIKM '03 Proc $12^{\text {th }}$ Intl Conf Inform Knowl Manag 556 (2003)

${ }^{56}$ S.C. de Lange, M.P. van den Heuvel, and M.A. de Reus, Neuroimage 141, 357 (2016)

${ }^{57}$ R.E. Brown and P.M. Milner, Nat. Rev. Neurosci. 4, 1013 (2003)

${ }^{58}$ W. Quattrociocchi, G. Caldarelli, and A. Scala, PLoS One 9, e87986 (2014)

${ }^{59}$ S. Prasad and L.D. Zuck, Proc. EXPRESS/SOS 30 (2016)

60T. Sun, P. Meakin, and T. Jøssang, Phys. Rev. E 49, 4865 (1994)

${ }^{61}$ M.E.J. Newman, Proc. Natl. Acad. Sci. USA 98, 404 (2001)

${ }^{62}$ X. Liang, J. Wang, C. Yan, N. Shu, K. Xu, G. Gong, and Y. He, PLoS One 7, e32766 (2012)

${ }^{63}$ A. Fornito and E.T. Bullmore, Eur. Neuropsychopharmacol. 25, 733 (2015)

${ }^{64}$ W. de Haan, K. Mott, E.C. van Straaten, P. Scheltens, and C.J. Stam, PLoS Comput. Biol. 8, e1002582 (2012)

${ }^{65}$ J.O. Maximo, E.J. Cadena, and R.K. Kana, Neuropsychol. Rev. 24, 16 (2014) 


\section{Captions}

Figure 1. Example calculation of graph $\hat{G}(1,1)$ from graph $G$, wherein each edge $\hat{g}_{i, j}$ is redefined as the sum of the connectivity between nodes $i$ and $j$ via all triangles spanning the rest of the network, e.g. $\hat{g}_{i, j}=g_{i, k_{0}} g_{k_{0}, j}+g_{i, k_{1}} g_{k_{1}, j}+g_{i, k_{2}} g_{k_{2}, j}$.

Figure 2. Relationship between quasi-idempotence and nodal strength heterogeneity, measured with respect to edge strengths variance, demonstrated through synthetic networks. a) When $\eta<<1$, edge strengths variance was closely determined by nodal strength heterogeneity: the initial matrix had a "striped" structure which was preserved by squaring, yielding high $t(1)$ and $t(\infty)$. b) When $\eta=1$, edge strengths variance was less closely determined by nodal strength heterogeneity: the matrix had a weaker "striped" structure, which was selectively enhanced by squaring, yielding intermediate $t(1)$ and $\imath(\infty)$. c) When $\eta>>1$, edge strengths variance was largely unrelated to nodal strength heterogeneity, thus squaring elicited a "striped" pattern not resembling the initial matrix, yielding low $t(1)$ and $t(\infty)$. d) and e) Accordingly, quasi-idempotence as assessed after one $(t(1))$ and infinitely many $(t(\infty))$ squaring iterations gradually decreased, in unison, with $\eta$. f) Near-perfect linear correlation was observed between $t(\infty)$ and the ANOVA $r_{\mathrm{ANV}}$-value representing the level of nodal strength heterogeneity, with $\iota(\infty) \approx \sqrt{2} r_{\mathrm{ANV}}$. For each matrix in a)-c), the colour-map was separately scaled between $5^{\text {th }}-95^{\text {th }}$ percentiles to aid visualization.

Figure 3. Quasi-idempotence of the emergent synchronization patterns formed by mean-field Kuramoto networks with noise. a) Average synchronization $\left\langle r_{i, j}\right\rangle$ increased with coupling strength $\lambda$ and decreased with noise intensity $\xi$, delineating regions of complete desynchronization, incomplete synchronization and complete synchronization. b) Standard deviation of the nodal strengths $\sigma\left\langle r_{i}\right\rangle_{j}$ was maximised in the region of incomplete synchronization, and c) ANOVA accordingly revealed maximum level of nodal strength heterogeneity. d) and e) The synchronization patterns featured varying levels of "deep" quasi-idempotence, with $t(1) \approx t(\infty)$, peaking in the region of incomplete synchronization in accord with $t(\infty) \approx \sqrt{2} r_{\mathrm{ANV}}$. f) and g) Initial and infinitely many-times squared matrices exemplifying emergent synchronization patterns that yielded high levels of "deep" quasiidempotence, (f) for $\lambda=0.26, \xi=0.41$ and (g) for $\lambda=0.64, \xi=0.74$; colour-map between $0.005-0.015$, matrices normalized to unit norm.

Figure 4. Quasi-idempotence of the emergent synchronization patterns formed by cycle amplitude fluctuations in an experimental ring of electronic chaotic oscillators. a) As a function of internal oscillator loop gain $G_{6}$ and coupling gain $G_{7}$, the average correlation dimension of the signals $\left\langle D_{2}\right\rangle$ delineated a "chaotic band", within which diverse synchronization patterns emerged. b) Average synchronization of amplitude fluctuations, measured as maximum time-lag cross correlation coefficient $\left\langle\max \left[C_{i, j}(\tau)\right]\right\rangle$, increased with coupling strength $G_{7}$ and was markedly heterogeneous within the "chaotic band". c) According to ANOVA, nodal strength heterogeneity tended to be maximised in regions of the "chaotic band" having intermediate or high average synchronization strength. d) and e) As revealed by $\imath(1)$ and $\imath(\infty)$, "shallow" quasi-idempotence was pervasively 
represented, but "deep" quasi-idempotence was manifest only for narrower control parameter regions. f) and g) Examples of "deep" quasi-idempotence $(t(1) \approx t(\infty))$, supported by islands of remote synchronization that introduced substantial nodal strength heterogeneity in the initial synchronization matrix. h) and i) Examples of "shallow" quasi-idempotence $(\imath(\infty)<<\imath(1))$, showing that substructures yielded by other types of remote synchronization, by cluster synchronization and synchronization gradients were dissipated during repeated squaring.

Figure 5. Quasi-idempotence of the emergent synchronization patterns formed by spontaneouslydeveloping networks of dissociated neurons. a) and b) The number of active electrodes (network size $N)$ and average coincidence index $\left\langle\mathrm{CI}_{i, j}\right\rangle$ increased steadily between 10 and 30 days in-vitro (DIV), reflecting the growth of new connections among the initially-dissociated neurons. c) The nodal strength heterogeneity (ANOVA $r_{\mathrm{ANV}}$ ) accordingly increased, most markedly between 10 and $20 \mathrm{DIV}$, settling on a high level afterwards. d) and e) As revealed by $t(1)$ and $t(\infty)$ changing in unison and closely tracking $r_{\mathrm{ANV}}$, the cultured networks spontaneously developed "deep" quasi-idempotence. f) Example synchronization pattern observed at DIV 10, demonstrating random-like structure which was dissipated by squaring. g) Example synchronization pattern observed at DIV 24, demonstrating a high-strength node and a large community, which conferred the network a moderate degree of quasiidempotence. h) and i) Example synchronization patterns observed at DIV 24 and DIV 34, demonstrating high nodal strength heterogeneity and correspondingly high degree of "deep" quasiidempotence. Colour-maps between 0.02-0.05 (f), 0.02-0.06 (g), 0.015-0.035 (h) and 0.01-0.03 (i), matrices normalized to unit norm.

Figure 6. Quasi-idempotence of the macroscopic structural and functional brain networks. a) Human structural connectivity, demonstrating high strength for nodes in the precuneus and posterior cingulate cortex: repeated squaring enhanced their preponderance and eventually dissolved all other substructures, revealing that the network had "shallow" quasi-idempotence $(t(\infty)<<t(1))$. Nodes 1499 left hemisphere, 500-998 right hemisphere, see Ref. (35) for labels. b) Human functional connectivity (synchronization of spontaneous activity), demonstrating subdivision into functional modules and higher strength of hub nodes principally in superior frontal, lateral frontal, and superior parietal regions; the difference between $t(1)$ and $t(\infty)$ was smaller, denoting a situation of "intermediate" quasi-idempotence. Node order follows default-mode, visual, fronto-parietal and central modules, see Ref. (37) for labels. c) Human co-activation across active tasks (Jaccard scores from literature meta-analysis), having structure closely correlated to synchronization of spontaneous activity, and accordingly "intermediate" quasi-idempotence. Node order as in (b). d) Mouse structural connectivity, with well-evident modular structure delineating the isocortex, pallidum-diencephalonmidbrain, and hindbrain; in this case, the network was relatively stable with respect to repeated squaring, revealing "deep" quasi-idempotence $(\iota(\infty) \approx t(1))$ in apparent contrast with human structural connectivity in (a). Nodes 1-148 left hemisphere, 149-296 right hemisphere, see Ref. (44) for labels. Colour-maps between 0-0.015 (a), 0-0.003 (b), 0-0.005 (c) and 0-0.005 (d), matrices normalized to unit norm.

Figure 7. Topographical distribution of nodal strengths over the human brain cortex before squaring (left) and after infinitely-many squaring iterations (right). a) Structural connectivity demonstrated high strength for nodes in the precuneus and posterior cingulate cortex, and repeated squaring had the 
effect of "focusing" nodal strength onto these regions. b) Functional connectivity (synchronization of spontaneous activity) and c) co-activation across active tasks (Jaccard scores from literature metaanalysis) by contrast yielded an initial nodal strength distribution which was appreciably more closely preserved by squaring, with high strength nodes principally in superior frontal, lateral frontal, and superior parietal regions. Blue-green-red colour-map and sphere radius represent rescaled nodal strength. 


\begin{tabular}{|c|c|c|c|c|c|c|c|}
\hline $\begin{array}{r}\text { Constituent } \\
\text { units }\end{array}$ & $\begin{array}{l}\text { Kuramoto } \\
\text { (phase) } \\
\text { oscillators (in- } \\
\text { silico) }\end{array}$ & $\begin{array}{l}\text { Non-linear } \\
\text { electronic } \\
\text { circuits (in- } \\
\text { silico) }\end{array}$ & $\begin{array}{l}\text { Neurons (in- } \\
\text { vitro) }\end{array}$ & $\begin{array}{l}\text { Neurons (in- } \\
\text { vivo, human) }\end{array}$ & $\begin{array}{l}\text { Neurons (in- } \\
\text { vivo, human) }\end{array}$ & $\begin{array}{l}\text { Neurons (in- } \\
\text { vivo, human) }\end{array}$ & $\begin{array}{l}\text { Neurons (in- } \\
\text { vivo, mouse) }\end{array}$ \\
\hline System nature & Simulated & Electronic & Biological & Biological & Biological & Biological & Biological \\
\hline Network scale & $\begin{array}{l}\text { Micro-scale } \\
(1 \text { osc./node })\end{array}$ & $\begin{array}{l}\text { Micro-scale } \\
\text { (1 osc./node) }\end{array}$ & $\begin{array}{l}\text { Meso-scale } \\
\left(\approx 10^{4} \text { neur. }\right. \\
\text { /node })\end{array}$ & $\begin{array}{l}\text { Macro-scale } \\
\left(\square 10^{6} \text { neur. }\right. \\
\text { /node })\end{array}$ & $\begin{array}{l}\text { Macro-scale } \\
\text { ( } \square 10^{6} \text { neur. } \\
\text { /node) }\end{array}$ & $\begin{array}{l}\text { Macro-scale } \\
\left(\square 10^{6} \text { neur. }\right. \\
\text { /node) }\end{array}$ & $\begin{array}{l}\text { Macro-scale } \\
\left(\square 10^{6} \text { neur. }\right. \\
\text { /node) }\end{array}$ \\
\hline $\begin{array}{r}\text { Link } \\
\text { definition }\end{array}$ & $\begin{array}{l}\text { Sync.(spontane } \\
\text { ous activity) }\end{array}$ & $\begin{array}{l}\text { Sync.(spontane } \\
\text { ous activity) }\end{array}$ & $\begin{array}{l}\text { Sync.(spontane } \\
\text { ous activity) }\end{array}$ & $\begin{array}{l}\text { Structural } \\
\text { connectivity }\end{array}$ & $\begin{array}{l}\text { Sync.(spontane } \\
\text { ous activity) }\end{array}$ & $\begin{array}{l}\text { Sync.(responses } \\
\text { to stimuli) }\end{array}$ & $\begin{array}{l}\text { Structural } \\
\text { connectivity }\end{array}$ \\
\hline $\begin{array}{r}\text { Underlying } \\
\text { signal }\end{array}$ & Phase & $\begin{array}{l}\text { Voltage } \\
\text { (continuous } \\
\text { amplitude } \\
\text { fluctuations) }\end{array}$ & $\begin{array}{l}\text { Voltage (inter- } \\
\text { spike intervals) }\end{array}$ & $\begin{array}{l}\text { Water self- } \\
\text { diffusion } \\
\text { anisotropy }\end{array}$ & $\begin{array}{l}\text { Blood oxygen } \\
\text { level-dependent }\end{array}$ & $\begin{array}{l}\text { Blood oxygen } \\
\text { level-dependent }\end{array}$ & $\begin{array}{l}\text { Water self- } \\
\text { diffusion } \\
\text { anisotropy }\end{array}$ \\
\hline $\begin{array}{r}\text { Form of } \\
\text { quasi- } \\
\text { idempotence }\end{array}$ & Deep & Deep, shallow & Deep & Shallow & Intermediate & Intermediate & Deep \\
\hline $\begin{array}{r}\text { Strength of } \\
\text { evidence }\end{array}$ & $\begin{array}{l}\text { Variable, } \\
\text { maximum for } \\
\text { intermediate } \\
\text { sync. level }\end{array}$ & $\begin{array}{l}\text { Variable } \\
\text { depending on } \\
\text { control } \\
\text { parameters }\end{array}$ & $\begin{array}{l}\text { Increasing with } \\
\text { culture age }\end{array}$ & $\begin{array}{l}\text { Strong, possibly } \\
\text { decreasing with } \\
\text { age }\end{array}$ & Strong & Strong & Strong \\
\hline
\end{tabular}

Table 1. Conceptual comparison of the in-silico, in-vitro and in-vivo systems taken into consideration, with corresponding form of observed network quasi-idempotence (self-similarity) and level of evidence. 


\section{Globally-coupled Kuramoto oscillators}
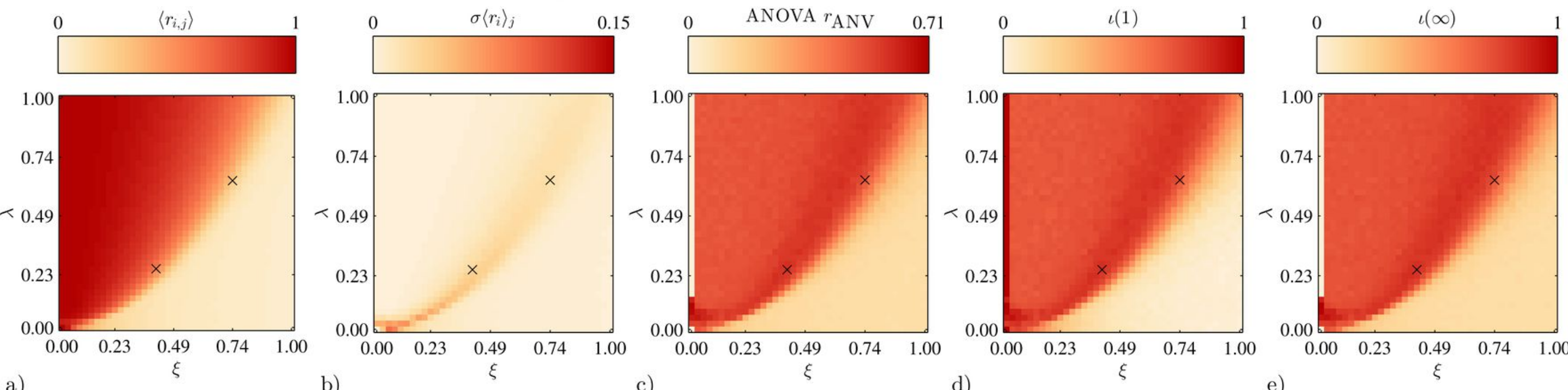

a)
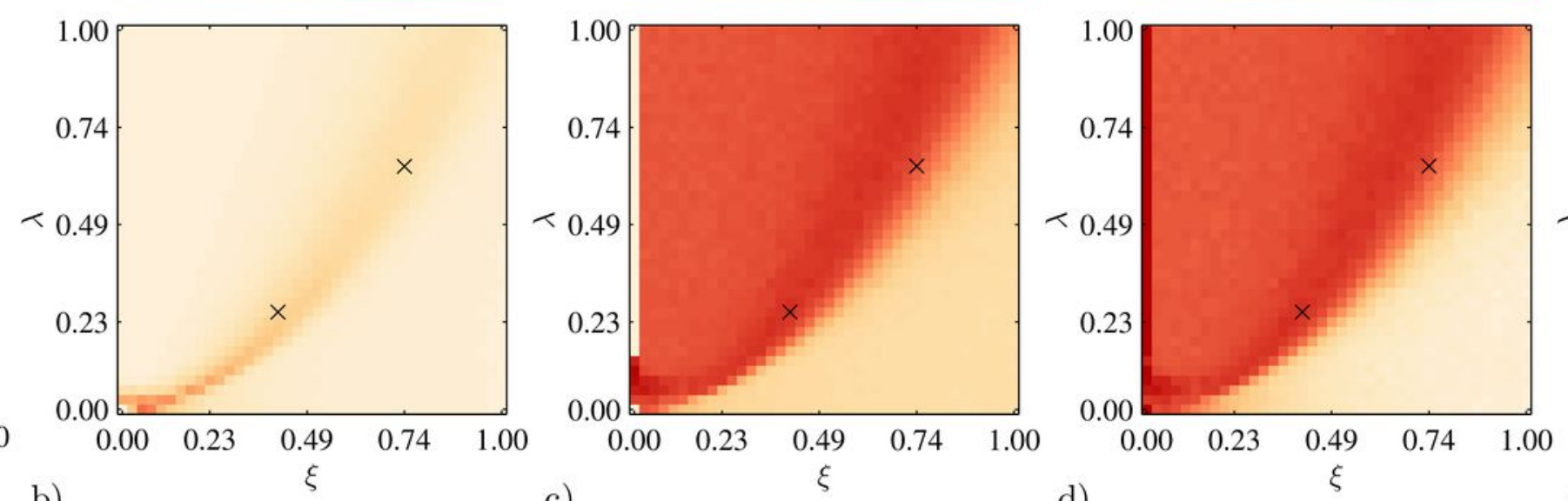

b)

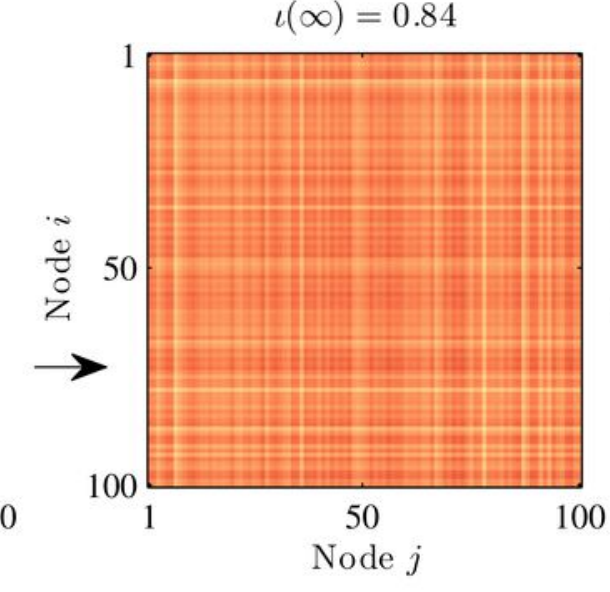

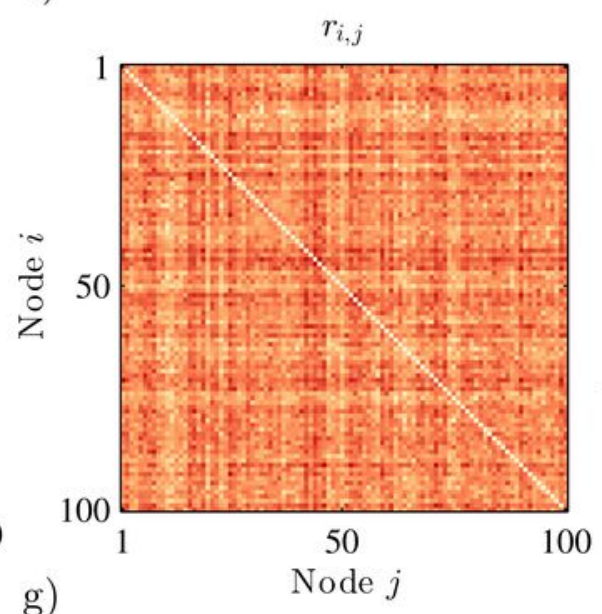

d)

$\iota(\infty)=0.74$

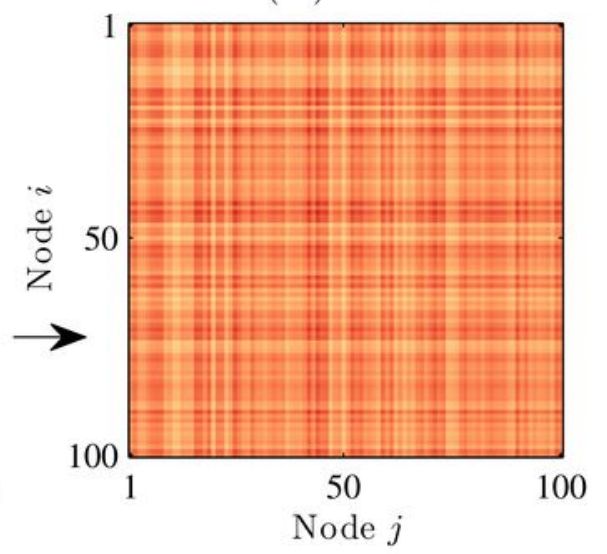
g) e)

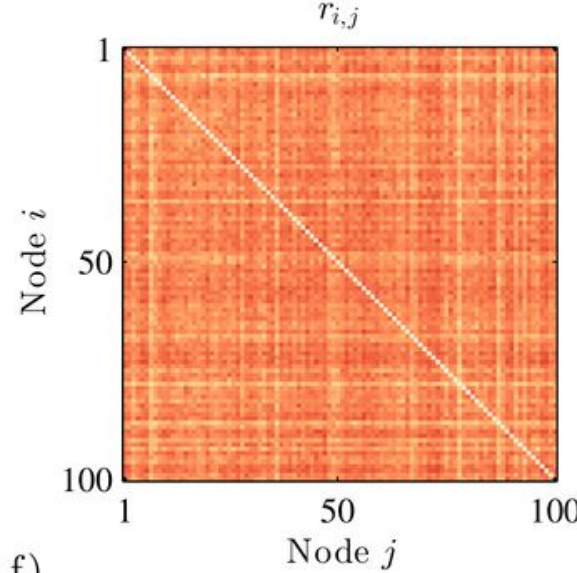


P-1 Experimental network of electronic oscillators
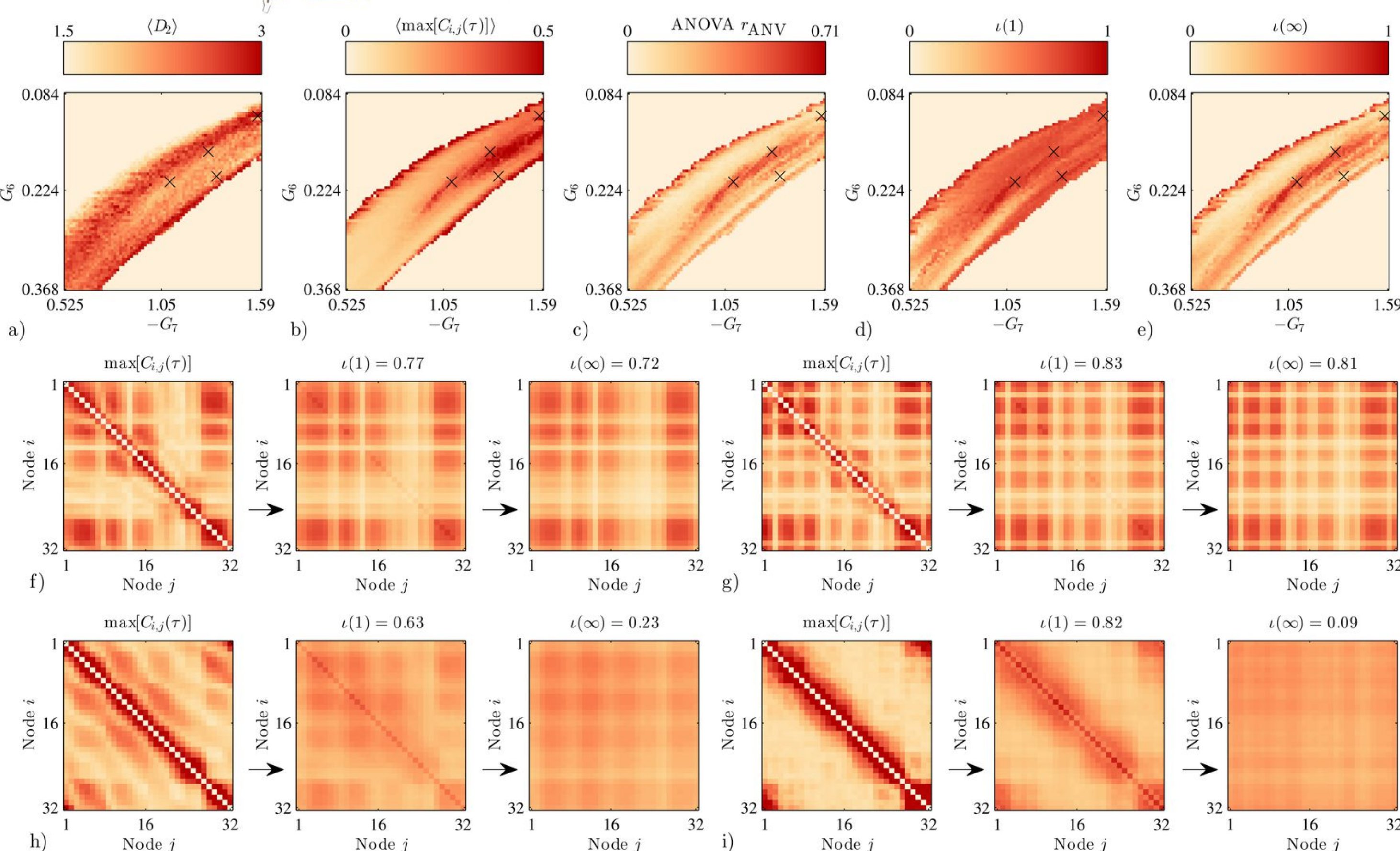


\section{- Networks of cultured dissociated neurons}

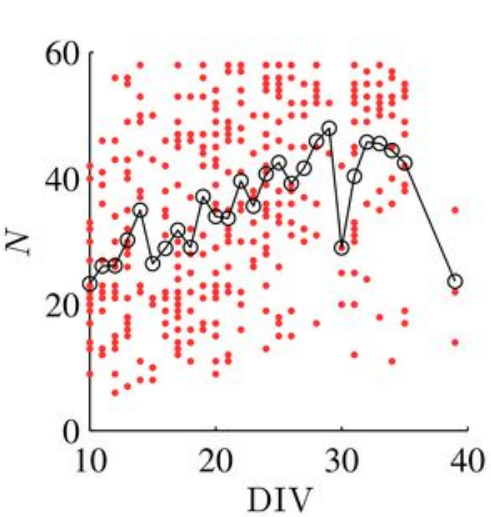

b)

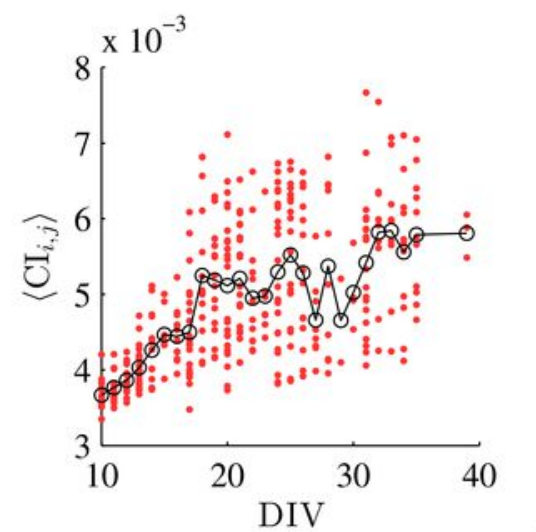

c)
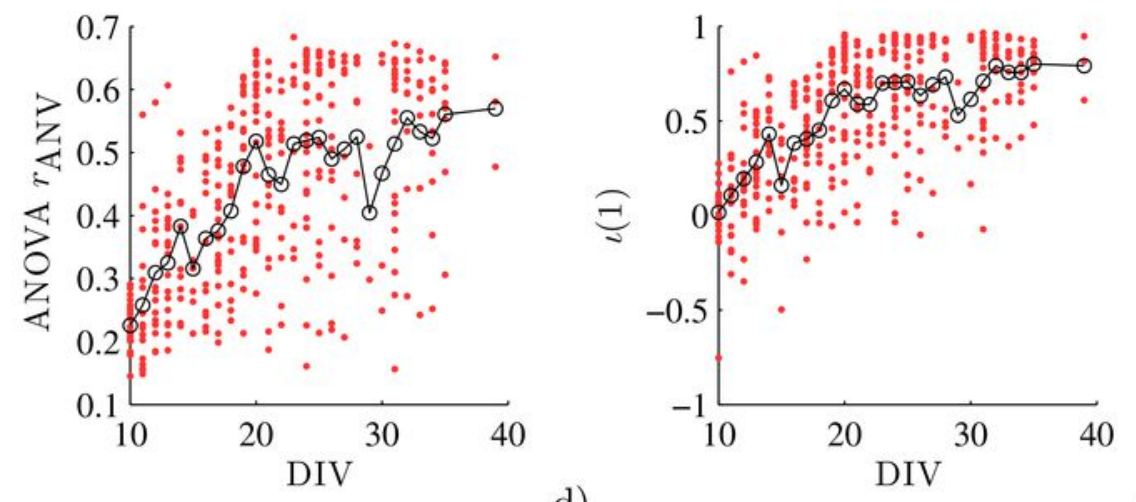

d)
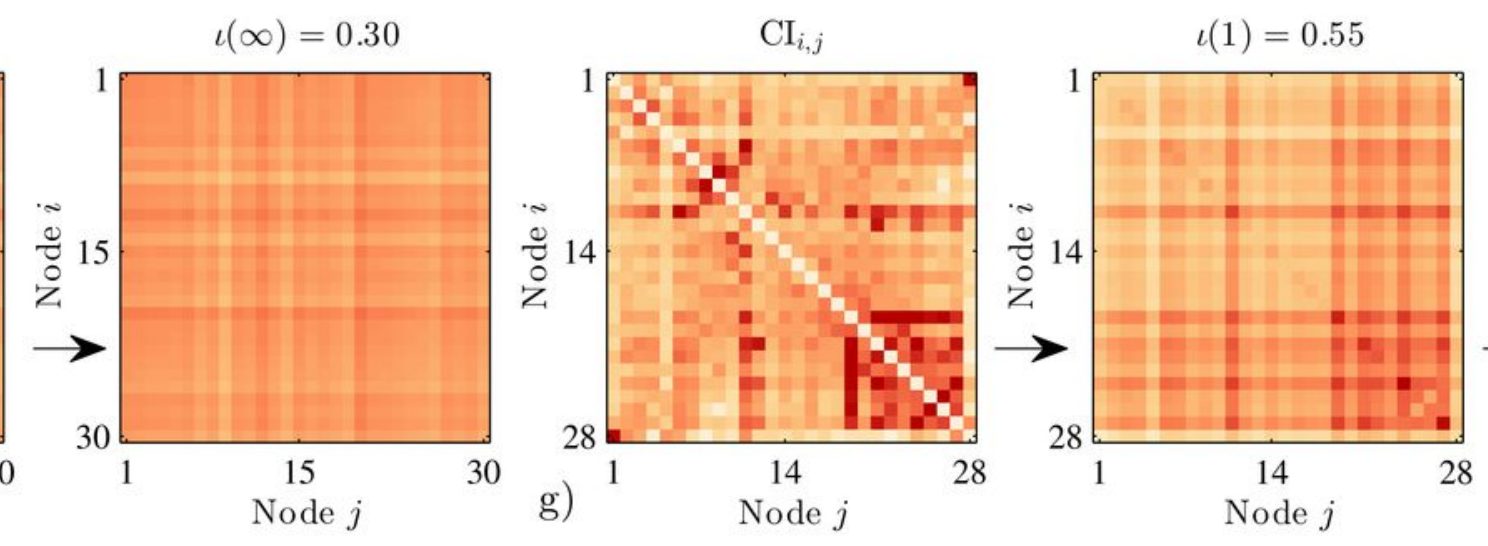

e)

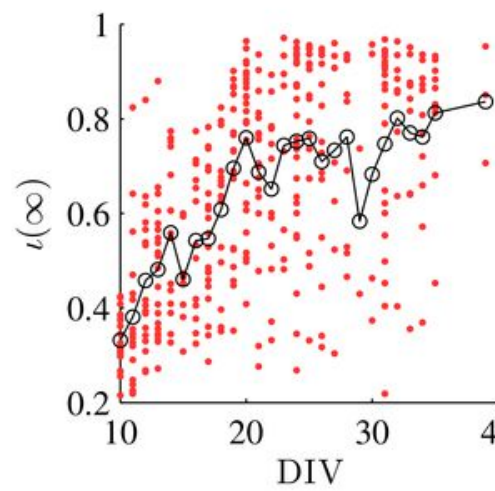

$\iota(1)=0.05$

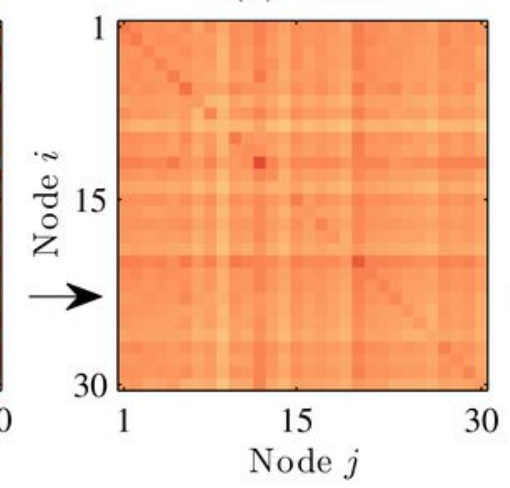

$\iota(1)=0.83$
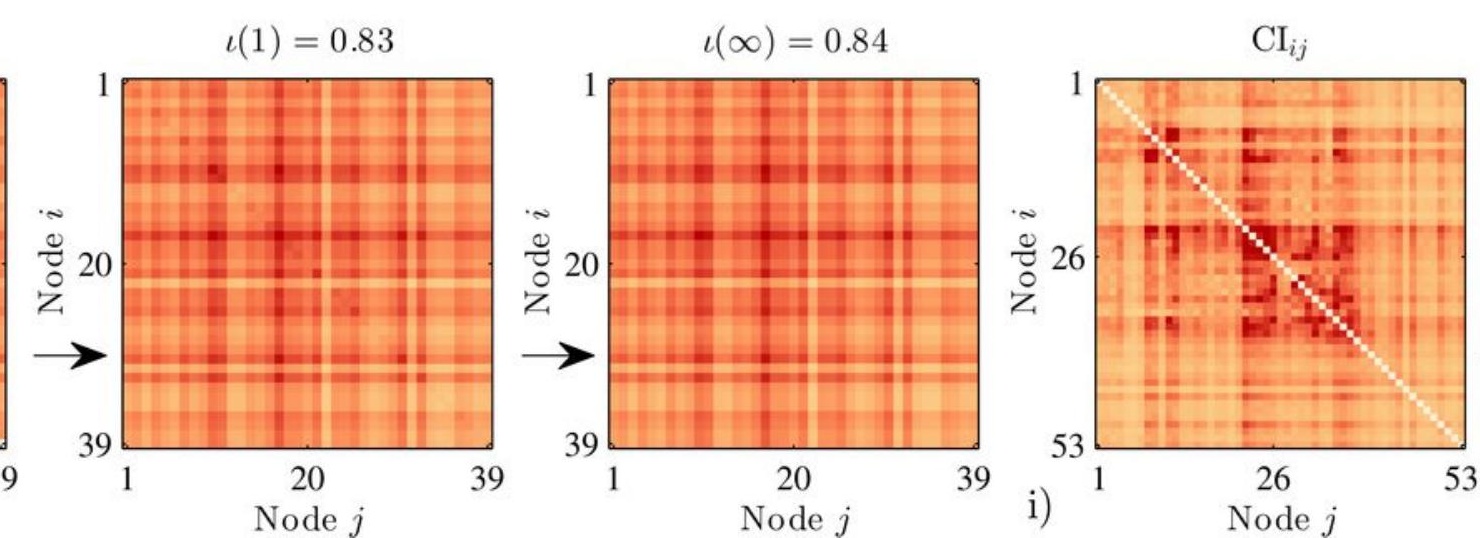

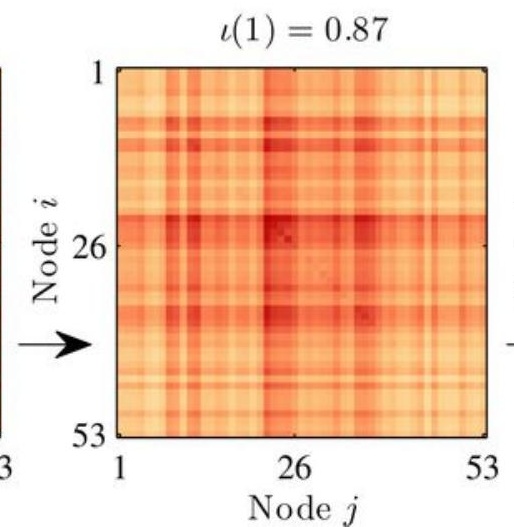

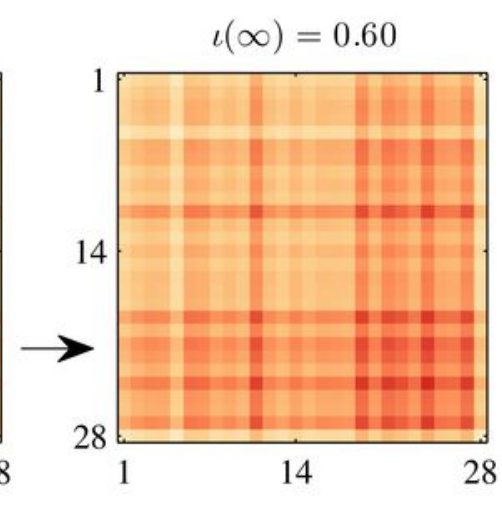

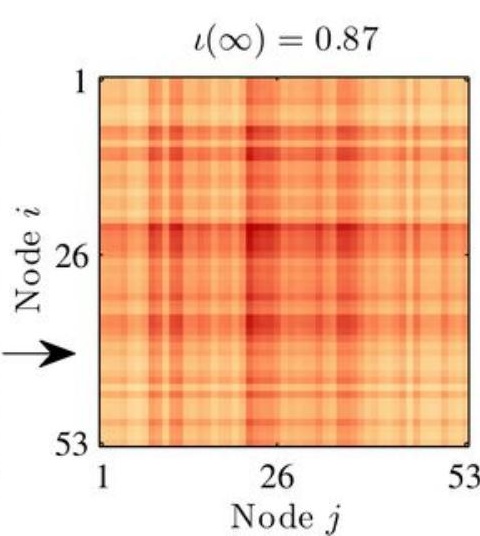



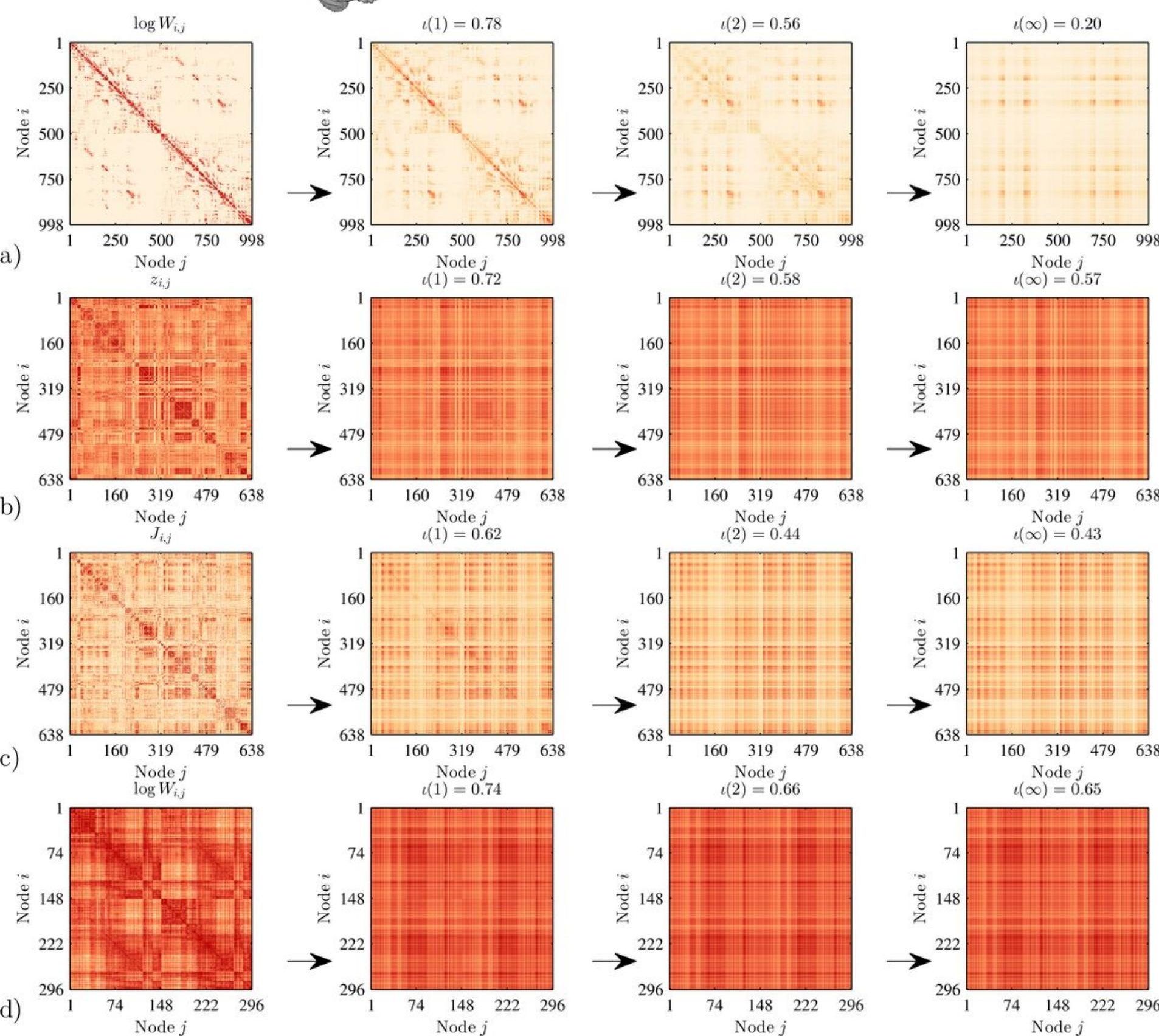
$\sum \log W_{i, j}$

$\iota(\infty)=0.20$

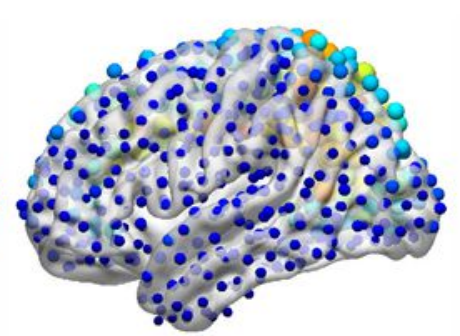

$\because 097390$

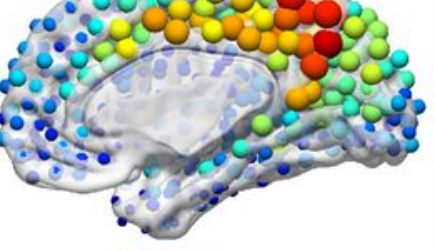

4080758

gene 03009

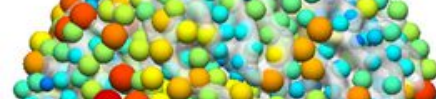
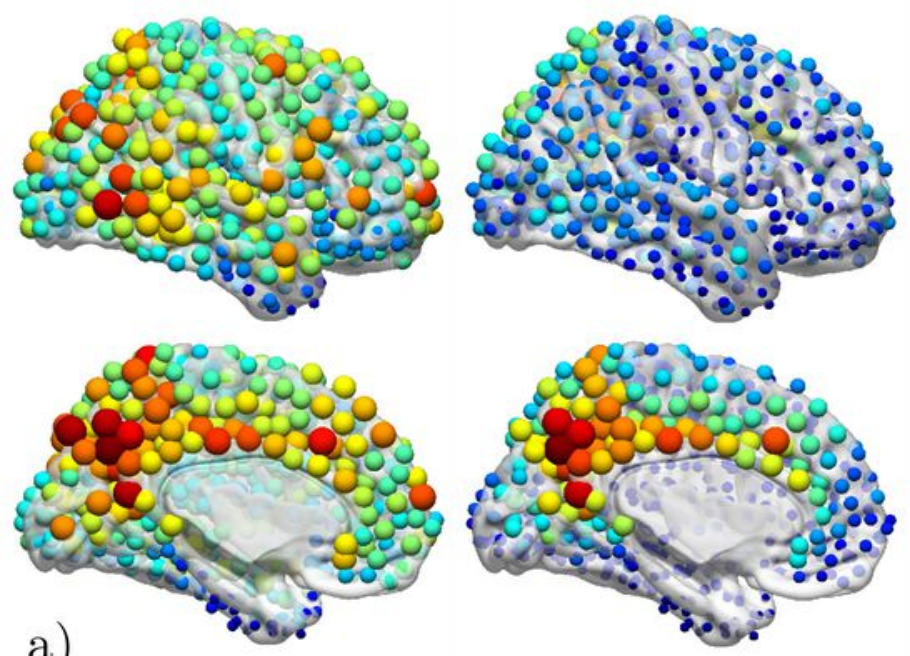

$\sum z_{i, j}$

$\iota(\infty)=0.57$

$\sum J_{i, j}$

$\iota(\infty)=0.43$
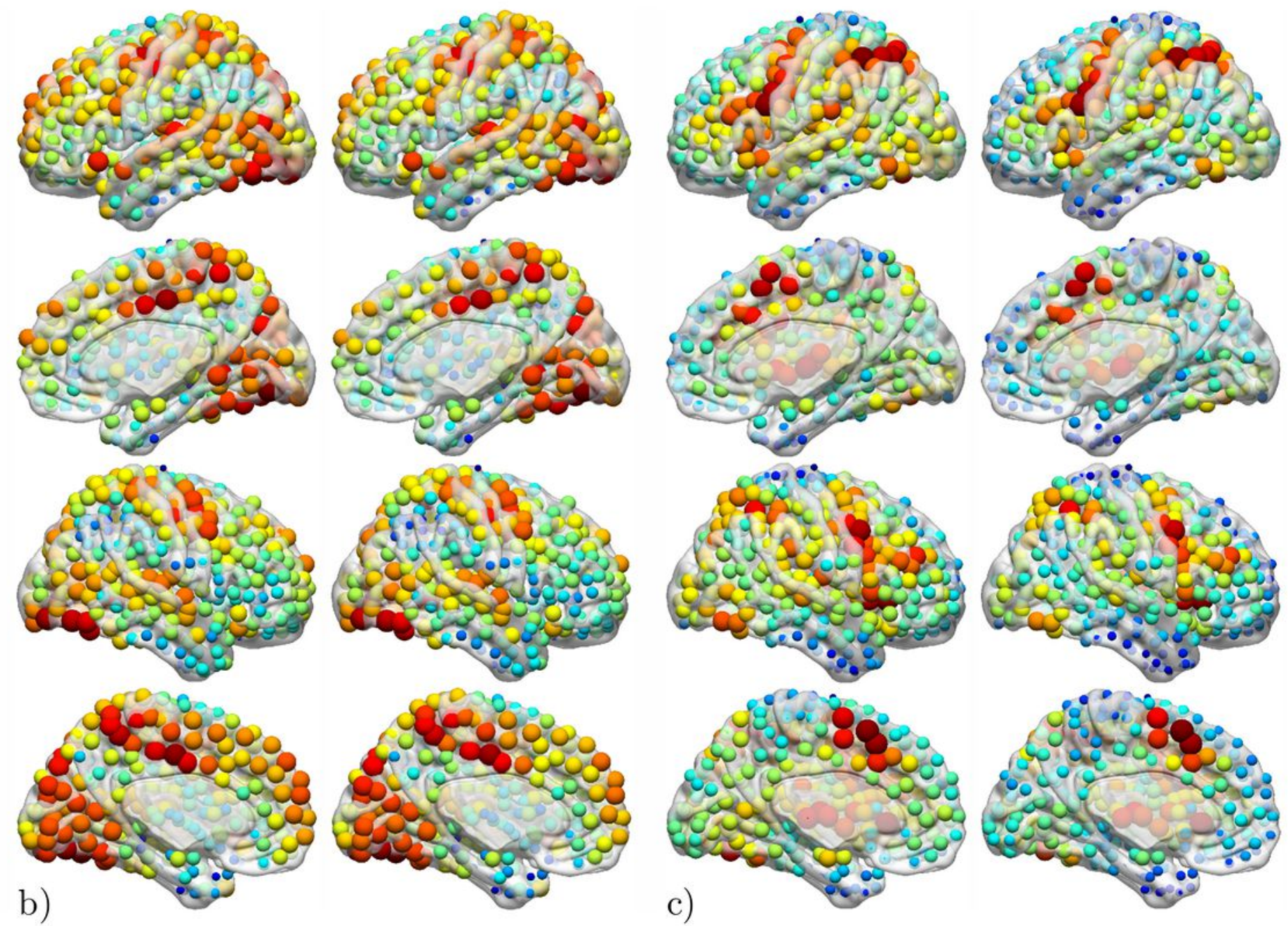